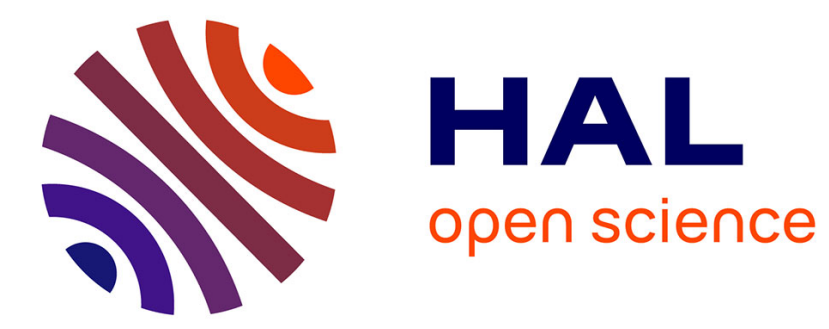

\title{
Une crise sociale en Chine: la société henanaise de l'été 1956 à l'été 1957
}

\author{
Jean-Luc Domenach
}

\section{To cite this version:}

Jean-Luc Domenach. Une crise sociale en Chine: la société henanaise de l'été 1956 à l'été 1957. Annales : Economies, Sociétés, Civilisations, 1979, 34 (5), pp.1069-1093. 10.3406/ahess.1979.294108. hal-01009176

\section{HAL Id: hal-01009176 \\ https://hal-sciencespo.archives-ouvertes.fr/hal-01009176}

Submitted on 17 Jun 2014

HAL is a multi-disciplinary open access archive for the deposit and dissemination of scientific research documents, whether they are published or not. The documents may come from teaching and research institutions in France or abroad, or from public or private research centers.
L'archive ouverte pluridisciplinaire HAL, est destinée au dépôt et à la diffusion de documents scientifiques de niveau recherche, publiés ou non, émanant des établissements d'enseignement et de recherche français ou étrangers, des laboratoires publics ou privés.

\section{(1) $(1) \Theta$}

Distributed under a Creative Commons Attribution - NonCommercial - NoDerivatives| 4.0 


\title{
Une crise sociale en Chine : la société henanaise de l'été 1956 à l'été 1957 \\ In: Annales. Économies, Sociétés, Civilisations. 34e année, N. 5, 1979. pp. 1069-1093.
}

\begin{abstract}
social crisis in China Henan society from summer 1956 to summer 1957 Jean-Luc DOMENACH Due to scarce and unreliable sources empirical research into the social situation Chinese province in 1956-57 can only yield very tentative conclusions The agricultural failure of 1956 accounts for the material hardships and economic indiscipline of the peasants Although envied and privileged urban life suffered from supply and housing problems as well as from the wide differences between cadres workers youth and occupational ethnic religious and political minorities In all however although discontent seems to have been widespread only minority actually made trouble So one can only speak of social crisis if we are prepared to admit that these popular disturbances constituted the maximum acceptable to political authorities who controlled the social body
\end{abstract}

Citer ce document / Cite this document :

Domenach Jean-Luc. Une crise sociale en Chine : la société henanaise de l'été 1956 à l'été 1957. In: Annales. Économies, Sociétés, Civilisations. 34e année, N. 5, 1979. pp. 1069-1093.

doi : 10.3406/ahess.1979.294108

http://www.persee.fr/web/revues/home/prescript/article/ahess_0395-2649_1979_num_34_5_294108 


\section{UNE CRISE SOCIALE EN CHINE : la société henanaise de l'été 1956 à l'été 1957}

Une des questions posées par l'historiographie de la Chine populaire concerne le retard des études sociologiques. S'explique-t-il par la nature des questions posées, par la définition chronologique des études ou plus simplement par l'absence de sources? Une recherche monographique, axée sur une province chinoise ${ }^{1}$ - le Henan, province centrale de par sa situation géographique et son destin historique - à une époque de changements critiques - la période de virage à droite économique, social et politique qui s étend entre l'échec agricole du mouvement des coopératives (été 1956) et le lancement du Grand Bond en avant (automne 1957) — recherche conduite à l'aide de sources d'origine provinciale ${ }^{2}$, ne permet pas de formuler une réponse définitive. Dune part, les sources sont plus riches que la presse centrale en faits de nature sociale. Elles indiquent, de façon claire, non seulement que la situation matérielle des Henanais a stagné ou empiré dans la période considérée, mais encore que la prise de conscience de cette détérioration a provoqué le mécontentement et une agitation sociale. Le premier secrétaire du Henan, Pan Fusheng, n’a-t-il pas déclaré au printemps 1957 : "Nous sommes assis sur un volcan ${ }^{3}$ ?

Est-il pour autant possible de définir cette situation par le concept de crise ? Si les sources contiennent des faits sociaux, elles ne permettent pas aisément de les qualifier. En effet, elles restent globalement insuffisantes et de valeur inégale selon les domaines. Surtout, mises bout à bout, les données dont nous disposons ne composent pas le tableau dramatique auquel fait référence Pan Fusheng. Il faut donc, à notre sens, reconstituer les principales caractéristiques de la société rurale et urbaine du Henan en 1956-1957 avant d'esquisser une définition spécifique de ce que peut être une crise sociale en Chine communiste.

\section{Le silence des campagnes}

C'est par les campagnes que la crise sociale de 1956-1957 est arrivée au Henan, c'est dans les campagnes qu elle eût pu ètre la plus dangereuse pour le régime : c'est pourtant là qu'elle fut la moins grave et en tout cas qu elle est la moins bien connue. La paysannerie est la grande muette d'un régime qui prétend avoir trouvé dans les campagnes sa légitimité première. Ce qui est vrai pour le Quotidien du peuple l'est tout autant pour le Quotidien du Henan : on n'y trouve pas un seul témoignage concret sur la vie paysanne. ses peines et ses joies, pas un seul reportage qui n’ait été écrit par un cadre ou dicté par des impératifs de 


\section{LA CHINE CONTEMPORAINE}

propagande. C"est pourquoi notre connaissance de la situation sociale des campagnes reste indirecte et imprécise.

A l'origine de la crise rurale, on trouve l'échec agricole provoqué par les excès et les maladresses du premier bond en avant imprimé à l'économie chinoise de l'été 1955 à l'été 1956. Il se résume globalement dans la stagnation de la disponibilité théorique de grains alimentaires par tête : $253,35 \mathrm{~kg}$ contre $253,25 \mathrm{~kg}$ en $1955^{4}$. Mais cette mesure est en grande partie fausse. Elle ne rend pas compte de l'appauvrissement réel de la population. Pour acheter du matériel, les coopératives avaient diminué les distributions en numéraire et même en céréales 5 . La reprise des activités secondaires et des échanges privés dans l'automne et l'hiver 1956, n'avait pas partout compensé les pertes de l'été. En second lieu. un paysage de catastrophe était soudain apparu sur plus de la moitie du territoire et l'on avait vu revenir, dans les zones les plus touchées par les inondations, des disettes, des paniques, des épidémies que l'on croyait à jamais disparues ${ }^{6}$. Nous ne pouvons malheureusement pas dresser un tableau précis des difficultés matérielles de la paysannerie. Nos sources se contentent dallusions à une situation que chacun connaissait de visu et par ouï-dire. Il est néanmoins certain que dans les zones frappées par les calamités, la population continua par endroits à manquer du nécessaire jusquà la récolte dautomne 1957. A Yuanyang, 17000 familles sur 70000 manquaient de grain au printemps ${ }^{7}$. Au début de juin, la situation alimentaire restait difficile sur 15 à $20 \%$ de la surface de la province. A la fin du même mois, dans 92 cantons de la région spéciale de Xinyang, la plupart des coopératives ne possédaient de réserves alimentaires que pour un mois. Des difficultés similaires sont signalées également dans le nord de la province, en juillet et en août 1957. Des paysans du Henan oriental poussés par la faim cherchaient à vendre leurs filles. Signe infaillible de xiahuang (disette d’été) : la réapparition dans certains endroits de la concurrence alimentaire entre l'homme et le bétail, qui provoqua des abattages, par exemple à Fengqiu, où le cours du porc sur le marché libre baissa de $60 \%$. Pendant l'hiver 1956-1957, les paysans manquèrent parfois plus encore de vêtements que de grain. En décembre, beaucoup vendaient leur ration alimentaire (kouliang) pour en acquérir. Bien que le gouvernement provincial eût distribue 600000 vêtements dans lautomne 1956, maintes localités en manquaient encore à la fin novembre. Des deux millions d'habitations detruites, beaucoup ne furent relevées que tardivement. Dans de nombreux endroits, la population se trouvait en mauvais état physique ${ }^{8}$. En septembre 1956, 83 districts étaient touchés par une épidémie de méningite. Dans la région de Nanyang, pourtant peu touchée par les inondations, 13000 personnes étaient atteintes de dysenterie et de malaria. Les inondations, la faim et la maladie provoquèrent des paniques que le pouvoir eut l'intelligence de canaliser dans de grands transferts vers les provinces désertiques du Gansu et du Qinghai ${ }^{9}$. Mais aux abords de Xinyang, dans le sud de la province, les paysans continuèrent pendant plusieurs mois à prendre d'assaut les trains pour aller mendier à Wuhan. Nous sommes cependant moins informés de la situation concrète des paysans dans les zones non sinistrées. Malgré de grandes variations locales, en 1956. le niveau de vie a stagné ou moins progressé que les années précédentes. La relance de l'artisanat et des activités privées, ainsi que la réouverture des marchés privés permirent une reprise progressive en 1957. Cette reprise produisit cependant un effet psychologique inattendu : elle confirma la masse des paysans dans le sentiment que la stagnation ou la baisse de leur revenu nétaient pas dues aux seules calamités naturelles, mais bien plutôt aux coopératives, aux erreurs des cadres et plus généralement à la politique du gouvernement ${ }^{10}$. Comme le proclamaient ironiquement des paysans de Yanshi, "dans le socialisme, tout est bon. même s"il n'y a rien à manger " ". Allait-on connaitre encore la misère pour avoir obéi aux folies de quelques-uns?

Ce qui a transformé en crise sociale l’appauvrissement de la minorité et la stagnation 
du niveau de vie de la majorité, c'est l'expression concrète de cette inquiétude : les retraits des coopératives. les attaques contre les cadres, et plus généralement lindiscipline économique.

Aux yeux des paysans. les coopératives portaient la responsabilité de léchec. La meilleure façon de s’en débarrasser, c'était évidemment de les quitter ${ }^{12}$. A cet effet, on pouvait déposer une demande officielle car les autorités, sans vraiment respecter le principe de l'adhésion volontaire, autorisaient désormais les départs dans certaines conditions. Une autre solution. la plus fréquemment pratiquée. consistait à se grouper pour récupérer les biens collectivisés, espérant ainsi imposer le fait accompli aux cadres. Des départs se sont produits dans de nombreux districts a au moins dix-neuf à notre connaissance. Ils commencèrent dans l'hiver 1956 et se poursuivirent jusque dans l'été 1957. Malgré les accusations officielles, ils n'ont pas concerné les seules catégories sociales dites "contre-révolutionnaires": dans les districts de Xinxiang. Linxian, Linru et Yingyang, la population "se ligua " pour quitter les cooperatives : 257 familles, soit la majorité des habitants de la coopérative Xianfeng (avant-garde) de Luoshan, voulaient retourner à l'agriculture privée. Nous connaissons au moins un cas de retrait de chef d'équipe de production qui n'a certainement pas èté unique si l'on en juge par les accusations plus générales de mollesse et de complicité qui ont été adressées à de nombreux cadres inférieurs et moyens dans l'automne 1957, quand la crise fut jugulée. Les effets de ces départs ne doivent pas être négligés. Ainsi, dans la région de Xinyang, ils contribuèrent à provoquer l'effondrement de 492 coopératives. Ailleurs, ils hâtèrent leur morcellement. Ils favorisaient plus généralement la formation dune atmosphère faite de disputes et de revendications.

Cependant, les départs effectifs n'ont concerné au total qu'une faible partie de la population rurale : 10000 familles dans la région de Xinyang qui en comptait plus d'un million, 8400 sur 100000 dans le district de Xixian. Le nombre des volontaires officiels au départ nétait pas beaucoup plus élevé : 200 familles dans un canton pourtant particulièrement troublè de Linru et 743 familles dans tout le district, 5800 sur environ 50000 dans le district de Tangyin, 1200 sur 30000 dans celui de Minquan. De plus. nombre de départs furent finalement temporaires. Si l'on admet les chiffres officiels de 40000 "foyers individuels" (dangan hu) en novembre 1957 contre 5572 en novembre 1956, seules 35000 familles environ auraient quitté les coopératives en un an ${ }^{13}$. Une explication de la faible ampleur du phénomène réside dans l'assouplissement volontaire de la politique officielle, notamment dans la division des coopératives et la relance des activités privées ainsi que dans la survivance purement nominale de maintes coopératives. dont le matériel était volé et les greniers pillés.

\section{Des troubles ou des incidents?}

La crise sociale, il est vrai, ne menaçait pas seulement les nouvelles institutions. Dans l'été 1957. la presse provinciale a soudain affirmé que les « ennemis de classe » avaient mis à profit l'agitation survenue dans les villes pour fomenter de véritables troubles contrerévolutionnaires à la campagne. Elle a présenté quelques exemples précis à l'appui de cette accusation ${ }^{14}$. Près de Zhengzhou subsistaient au printemps 1957 plusieúrs sociétés bouddhistes illégales ainsi qu'un " parti de l'égalité » dont nous ne savons rien. Un " parti du genre humain " recrutait parmi les lycéens de Shenxian et un " parti de l'univers " près de Jiaozuo. Deux organisations subversives ont été écrasées à grand fracas de publicité. La "Société des prosternants ", déjà dissoute en 1953, comprenait encore 120 adhérents dont certains infiltrés dans des échelons locaux du parti communiste et de l'administration. Elle poursuivait ses activités dans cinq districts (Mengxian, Gongxian, Yingyang, Kaifeng et Tongxu) et disposait de dix fusils, 270 cartouches et... 291 dollars américains. Le " parti 


\section{LA CHINE CONTEMPORAINE}

des jeunes catholiques de la nouvelle Chine ", fondé au printemps de 1956, comptait une centaine de membres dispersés dans les districts montagneux des deux régions administratives de Nanyang et de Xiangyang (Hubei). Bien qu'on lui ait prêté des projets de coups de force, son activité etait principalement politique : on na saisi sur ses membres qu un fusil et trois revolvers, mais de nombreuses cartes d'adhésion et des documents dénonçant la politique rurale du régime. Ces exemples ne manquent pas dintérêt. Ils rappellent que le nouveau régime n’a pas pu écraser d'un coup les sociétés secrètes ni empêcher que subsistent, surtout après les mauvaises récoltes. des zones mal contrôlées où de petits groupes dopposants poursuivaient des activités épisodiques. Cependant, ils ne suffisent pas à prouver l'existence de véritables troubles politiques. Aucune des organisations démantelées dans l'été 1957 n'exerçait ses activités à l'échelle de toute la province. La subversion qui leur fut reprochée etait de nature principalement idéologique : aucune navait les moyens et probablement même l'intention de passer aux actes.

En fait, la crise rurale a donné lieu à des incidents localisés et sporadiques ${ }^{15}$ plutôt quà de véritables troubles politiques.

Ces incidents ont fréquemment opposé les paysans aux cadres de base. Il arrivait que les paysans se contentent de ridiculiser et de railler leurs dirigeants. Mais des menaces précises succédèrent parfois aux quolibets : à l'époque du partage de la récolte d'été. le comptable d'une coopérative du district de Yanshi n'osait plus rentrer chez lui de peur de se faire écharper. Ces menaces furent au moins une fois suivies d'effet : dans un village de Shangcheng, trois enfants de cadres furent assassinés.

La presse a voulu faire croire que ces incidents avaient été les plus dangereux en juinjuillet, au moment où la direction provinciale hésitait sur la réponse à opposer aux accusations des droitiers. Ainsi, "mille slogans réactionnaires" seraient apparus sur les murs d'une coopérative du district de Xinyang dans la nuit du 31 juillet au premier août 1957 ; et à Yinyang un ancien propriétaire foncier proclama à cette époque « qu il y aurait une révolte en août $»$. Cette datation n'est pas invraisemblable, puisque les mois de juillet et août furent le moment où l'on répartit une récolte d'été souvent décevante. Ainsi, les désordres de Yanshi, qui durèrent vingt jours, furent provoqués par une baisse de la production de blé. Notons cependant que plusieurs autres sources situent les incidents avant et non après la récolte de blé. De toute façon, celle-ci s’avéra rarement catastrophique, et elle améliora en fait souvent la situation alimentaire. Notre impression est que l'agitation a été endémique, avec une pointe peut-être dans l'été 1957.

En revanche, l'attribution de la responsabilité des incidents aux seuls " propriétaires fonciers, paysans riches, mauvais éléments et paysans moyens aisés " parait très contestable, encore que des cas de ce type se soient présentés. A Xincai, on tenta de dérober le registre des individus placés sous surveillance. Dans un canton de Minquan, sept anciens brigands et treize membres de sociétés secrètes auraient conduit lagitation. Certains anciens propriétaires fonciers n'auraient pas hésité à reprendre par la force une partie de leur bétail ou de leurs terres ${ }^{16}$. Parmi les 9159 familles de propriétaires fonciers et de paysans riches de Linxian (qui comportait environ 110000 foyers), $20 \%$ se seraient mal comportées jusquà l'èté 1957 ainsi que $71 \%$ des 263 familles de "contrerévolutionnaires " et $22 \%$ des 22990 familles de paysans moyens aisés. Dans l'ensemble de la province, trois mille membres de ces catégories sociales devaient être passés en jugement en septembre et octobre 1957. Mais on se demande bien comment les autres catégories de la population rurale, qui étaient également impliquées dans les retraits des coopératives et, comme nous le verrons, dans les délits économiques, ne se sont pas rendues coupables de tels crimes. La presse elle-même devait insister à diverses reprises, en septembre-octobre 1957, sur le mauvais moral de nombreux paysans pauvres et « moyens pauvres ". D’après Pan Fusheng, lui-même, la proportion des "éléments attardés " (c'està-dire des mécontents) dans six coopératives était, au début de l'automne 1957, de $8 \%$ 
parmi les " anciens paysans moyens-pauvres ", de $5.3 \%$ parmi les « moyens-pauvres » et de $4.2 \%$ chez les paysans pauvres; et la proportion de "centristes ", c'est-à-dire d'hésitants, était respectivement de $40 \%$, de $28 \%$ et de $23 \%$. Sur les 124 familles coupables de "graves tendances capitalistes" $(8.9 \%$ de la population des six coopératives), 64 appartenaient à ces trois catégories. $\mathrm{Au}$ total, les informations disponibles nous portent à la prudence. Les campagnes du Henan n'ont pas connu de mouvements de rébellion, mais quelques troubles très localisés. Plus fréquents semblent avoir été les incidents de nature diverse et finalement peu graves. Ces incidents témoignaient d'une hostilité très répandue à l'égard des cadres.

\section{L'indiscipline économique}

Les paysans profitèrent, en effet, des appels officiels à dénoncer les excès dont avaient pu se rendre coupables leurs dirigeants locaux. Les " assemblées des représentants des membres des coopératives " dont les autorités supérieures encourageaient la convocation ne pouvaient pas toujours être complètement manipulées et contrôlées par les cadres locaux ${ }^{17}$, et il arrivait que de graves critiques y fussent exprimées. Les plus nombreuses concernaient l'autoritarisme. les brutalités et les avantages des cadres ${ }^{18}$. En butte à la méfiance et à l'hostilité de la population rurale, les cadres ne se trouvaient plus autant soutenus que dans le passé par leurs supérieurs. Ceux-ci les rendaient aujourdhui responsables d' "erreurs" qui, lors du mouvement de collectivisation de 1955-1956, étaient des " expériences avancées ». Leur pouvoir avait été diminué par la division des coopératives. la redistribution des lopins de terre et la réouverture des marchés privés. La politique rurale avait changé à leur désavantage et sans qu iils fussent consultés. Aussi, par crainte ou par conviction, ils hésitaient désormais à affirmer leur autorité. De nombreux articles de presse ont déploré l'affaiblissement de lautorité des cadres ruraux ${ }^{19}$. Le plus souvent, ceux-ci oubliaient d'appliquer complètement les mesures impopulaires qui fixaient les limites de la libéralisation. Parfois aussi, leur désillusion était moins passive. A Yanshi, dans le canton de Fudian, 7 membres du Parti rendirent leur carte et 47 cadres renièrent publiquement les objectifs politiques du régime ${ }^{20}$. Il arrivait que les cadres de base prissent fait et cause pour les paysans. A Jiyuan, 8.6\% des cadres exerçaient des " activités capitalistes "; dans les quatre districts de Linru, Yingyang, Xinxiang et Linxian, $20 \%$ des cadres se montraient " passifs "; et, sur les 11121 cadres que comptait le district de Linxian, 1409 (soit 12.6\%) étaient classés comme "opportunistes de droite ", 741 $(6,7 \%)$ manifestaient " une idéologie et des activités capitalistes », $1040(9.5 \%)$ avaient commis des erreurs " localistes " et $555(4,9 \%$ ) avaient fait preuve d" "individualisme " : ce qui, en clair, signifiait qu'une importante proportion des cadres estimaient qu'il fallait durablement interrompre le processus de collectivisation, se livraient à des trafics divers ou les autorisaient ${ }^{21}$. Soudain en veine dexplications idéologiques et de statistiques invérifiables, la presse devait, à l'automne 1957, attribuer cette situation aux défauts " petits-bourgeois " intrinsèques à la condition paysanne $(80 \%$ des cadres ruraux étaient d'extraction villageoise) et au rôle de vecteur du capitalisme dans les campagnes que jouaient les " paysans moyens aisés ", c'est-à-dire les paysans moyens qui s’étaient enrichis depuis la Libération. Quoi qu'il en soit, l'affaiblissement de l'autorité des cadres et fréquemment leur compromission active ont ouvert la voie à la manifestation la moins contestable et la plus importante de la crise sociale dans les campagnes henanaises: l'indiscipline économique ${ }^{22}$. Parmi 371 secrétaires de coopérative dans le district de Shangcheng, 144 avaient, au cours de l'année 1957, accepté ou conduit d'eux-mêmes des dissimulations et des partages de production illicites. Cette indiscipline économique s'est d'abord traduite par la négligence du travail collectif et des plans de culture. Un peu partout, les paysans quittaient à tout moment leur équipe de production pour aller 


\section{LA CHINE CONTEMPORAINE}

chercher ailleurs un travail plus rémunérateur. Dans les zones cotonnières (oủ l'on n'avait pas oublié les retards des livraisons de grains dans l'automne 1956), ils réclamaient que l'on donnât la priorité aux cultures céréalières et que l'on réservât les terres médiocres au coton. Malgré les admonestations publiquement adressées aux districts coupables, la province ne put finalement semer que 800000 hectares de coton, souvent sur de mauvais sols, au lieu des 900000 initialement prévus. Les actes d'indiscipline les plus graves concernaient le système d'achat et de vente unifié par lequel l'État contrôlait, depuis 1953 , la commercialisation des principaux produits agricoles. Les paysans voulaient recevoir le plus possible et livrer le moins possible de grains. Prétextant des difficultés exceptionnelles et se sentant encouragés par l'attitude plus indulgente des autorités, les coopératives multipliaient les demandes de grains alimentaires (kouliang), que celles-ci fussent ou non justifiées. Ainsi, 90 tonnes de grains supplémentaires furent réclamées et obtenues par cinq cantons de la région de Shangqiu. Au total, l'État dut livrer aux campagnes (ainsi quaux villes où des réclamations analogues se faisaient entendre) plus que précédemment et plus que prévu : 2,4 millions de tonnes entre l'automne 1956 et l'été 1957, soit $48 \%$ de plus que l'année précédente et 250000 tonnes de plus que prévu.

Or, dans le même temps, les opérations de collecte du grain par l'État se déroulaient plus difficilement et plus lentement que d'ordinaire. Pour diminuer le nombre des livraisons à un tarif dérisoire, par rapport aux prix pratiqués sur le marché libre. les coopératives faisaient de fausses déclarations et dissimulaient une partie de leur production. Ainsi, à l'automne 1956, le district de Pingxing n'avait déclaré qu'une récolte annuelle de céréales s'élevant à 723 tonnes au lieu des 875 tonnes effectivement récoltées. Après la récolte de l'été 1957 , les autorités estimèrent à $10 \%$ la part de la production dissimulée. De plus, une fois livré leur quota, les coopératives excédentaires rechignaient à vendre au cours officiel la part réglementaire $(60$ puis $80 \%)$ de l'accroissement de production. Les membres des coopératives se partageaient le plus souvent ce qui était soustrait aux réquisitions officielles. 95 tonnes de grains furent ainsi distribuées à l'intérieur de 35 coopératives de Huixian dans l'été 1957. Une partie était consommée et lautre commercialisée sur les marchés proches. Au début de septembre 1957, 150 tonnes de patates douces étaient vendues chaque jour sur les marchés locaux du district de Neihuang. Les partages sauvages de coton étaient au moins aussi répandus, car les paysans le revendaient au marché noir ou bien l'ouvrageaient à domicile. Ainsi, dans le district de Taikang, 350 tonnes de coton furent écoulées sur le marché noir à la fin de 1956.

Ces partages étaient souvent effectués publiquement à l'intérieur des coopératives avec l'accord actif des cadres. Maintes cooperatives de la banlieue de Zhengzhou, sous la direction de responsables habiles, se transformèrent en de véritables entreprises commerciales qui, négligeant les contrats officiels passés avec les compagnies d'État. traitaient directement, à des tarifs très élevés, avec les petits marchands de la ville et même avec des cantines d'entreprises publiques. La presse nationale monta en épingle le cas d'une coopérative du district de Runan qui avait conclu des contrats de fourniture de chanvre et de cordes dans plus de vingt villes chinoises dont Pékin, Tientsin, Shenyang. Shijiazuang et Zhengzhou: au début de l'été 1957, cette coopérative particulièrement entreprenante avait déjà réalisé un profit de plus de 20000 yuan.

A cause de cette multitude d'actes d'indiscipline locaux, les opérations dachat par l'État des récoltes de 1956 et de la récolte de 1957 ne purent être achevées à temps. A la fin de novembre 1956, le plan d'achat des céréales pour lensemble de la province n'était rempli quà $77,9 \%{ }^{23}$. Il fallut plus de temps encore pour collecter le coton et les oléagineux. A la fin de février 1957, la région de Shangqiu navait toujours livré que $35,2 \%$ de son quota de coton pour 1956. Le plan dachat de coton ne fut probablement pas accompli non plus en $1957^{24}$ 
Il faudrait pouvoir résumer l'attitude de la paysannerie henanaise des années 19561957 en traçant le tableau de deux paysans. Le premier serait l'un de ces paysans sinistrés en 1956, tout juste secouru pendant l'hiver et qui n’a échappé à la faim quaprès la récolte de l'été ou de l'automne 1957. Ce portrait, nous ne sommes pas en mesure de le faire. Les paysans de ce type étaient nombreux, nous le savons, mais ils navaient pas droit à la parole et moins encore les moyens de la prendre. Leur silence, c'est le silence de la misère. d'une misère qui ne compte pas. En revanche, nous pouvons esquisser le portrait d'un autre type de paysan. Il n'est pas riche, mais il a les moyens d'échapper à la misère. Voici donc Shen Xiaozhang. du district de Changge. Chef d'une famille de 11 personnes, il avait reçu au total $3500 \mathrm{~kg}$ de grains en partage pendant lannée 1956. De plus, arguant de ses charges familiales, il avait su obtenir des cadres de sa cooperative des rations supplémentaires au titre des " secours", ce qui avait incité les autres paysans du village à en réclamer aussi. Pour augmenter ses revenus, il envoyait régulièrement deux de ses fils vendre du grain et des légumes sur le marché voisin et lui-même se livrait à l'usure, prêtant du soja à $30 \%$ d'intérêt sur trois mois ${ }^{25}$. Comme il refusait de lui donner $100 \mathrm{~kg}$ de grains, sa belle-fille le dénonça aux autorités en février 1957. Une enquête découvrit chez lui une réserve de $3710 \mathrm{~kg}$ de céréales diverses. Mais les cadres locaux, sachant que son cas n'était pas isolé, se contentèrent d'exiger la restitution des subsides indûment perçus et ce n'est qu'en août qu'il dut cesser ses activités ${ }^{26}$. Shen Xiaozhang, c'est la paysannerie qui revendique et qui combine - c'est la paysannerie qui, depuis l'été 1956, inquiète le régime.

Reste que de telles revendications et manœuvres sont demeurées d'ordre principalement économique et qu'à l'exception d'incidents localisés elles n'ont jamais pris un tour politique. On retrouve là une énigme majeure qui traverse toute lhistoire de la Chine populaire : bête de somme du régime, la paysannerie ne s'est pas insurgée contre le sort misérable qui lui était réservé. Elle a simplement tenté de survivre ou, comme Shen Xiaozhang, daméliorer temporairement sa condition.

\section{Crise de la société urbaine}

Jusque dans l'èté 1957, les villes du Henan présentent la même physionomie sociale d'ensemble que les campagnes : alors qu'une minorité s'agite et revendique, la majorité se contente de tirer profit silencieusement de la nouvelle atmosphère. Leur situation comporte cependant deux particularités majeures: tout d'abord, quoique également marginale, l'agitation revêt bien plus de gravité dans les villes. Elle est numériquement plus importante, géographiquement plus concentrée. Surtout, elle apparait plus dangereuse, car elle se produit dans le lieu mème $\cdots$ la ville - où le nouveau pouvoir est installé, à quelques centaines de mètres des administrations provinciales.

En conséquence, la presse provinciale en dit beaucoup plus sur la situation des villes que sur celle des campagnes. Le Quotidien du Henan, c'est en fait le journal de la province vue de Zhengzhou. Destiné d'abord aux cadres et aux militants du Parti, en majorité citadins reels ou potentiels, il leur parle surtout de ce qui les intéresse le plus : leurs villes. Cette publicité des événements urbains, qui devait concourir au retentissement des Cent Fleurs. facilite la tâche de l'observateur : elle lui permet d'esquisser une description de la société urbaine du Henan en 1956-1957.

\section{La vie rèvée}

Une société s'organise tout autant en fonction de ses mythes que de sa réalite. En l'occurrence, mythes et réalité s'accordent: vivre en ville. c'est mieux. Comme on les 


\section{LA CHINE CONTEMPORAINE}

interrogeait sur leurs ambitions professionnelles, voici ce que répondirent les élèves d'une classe de deuxième année du lycée de Changge, un district rural : 18 voulaient devenir cadres, 7 officiers, 7 " spécialistes" (zhuanjia), 2 aviateurs, 2 ouvriers, 2 professeurs et 8 seulement paysans. Les professions de nature urbaine recevaient donc une nette préférence $^{27}$. Tout confirme lattrait exercé par la ville sur les jeunes gens ${ }^{28}$. S'ils cherchent à poursuivre leurs études et à se faire embaucher, c'est aussi pour s'y glisser ou y demeurer. Les filles de la campagne font la chasse aux cadres et ouvriers à marier, au risque d'ailleurs de ne pas s'acclimater et d'être finalement rejetées par un compagnon qui les trouvera trop rustres ${ }^{29}$. La ville attire aussi les moins jeunes comme les ouvriers temporaires qui espèrent la régularisation de leur statut et comme ces paysans sinistrés qui s.y infiltrent pour exercer des petits métiers. Nos sources ne permettent malheureusement pas d'évaluer l'importance numérique de l" "immigration noire". Il se peut que les grosses bourgades du Henan. souvent peu dégagées de leur environnement rural, aient exercé un moindre attrait sur la paysannerie que les métropoles de Chine de l'Est ou du Sud ${ }^{30}$. Mais aussi bien les avertissements répétés de la propagande officielle que les plaintes des paysans sur l'inégalité des conditions ${ }^{31}$ et les faits mentionnés plus haut laissent peu de doutes sur l'existence de ce phénomène.

Les mythes avaient raison. La vie était incontestablement meilleure en ville. Tout d'abord, les citadins jouissaient d'un revenu bien plus élevé et plus stable que les paysans. D’après un directeur adjoint du Bureau de la statistique du gouvernement provincial, le salaire ouvrier moyen atteignait 498 yuan par an, soit, selon lui, en tenant compte des avantages en nature dont bénéficiaient les paysans, $180 \%$ de plus que le revenu des membres des coopératives rurales ${ }^{32}$. Cette comparaison, en fait, n'avait guère de sens. En effet, quoi de commun entre la subsistance toujours difficile et menacée du paysan et la rémunération monétaire régulière dont bénéficiaient la plupart des citadins? Même si tous les salaires urbains nètaient pas aussi élevés que ceux des ouvriers, même si certaines catégories de citadins connaissaient, comme nous le verrons, une existence précaire, pour la grande majorité, vivre en ville, c’était vivre en sécurité. En outre, grâce aux écoles du soir et à la proximité des centres de décision, on pouvait gravir les échelons et, surtout, se glisser plus vite dans le Parti. Vivre en ville, c'était aussi pouvoir espérer dans l'avenir. Cette vie que les autres leur envient, les citadins la ressentent pourtant comme une vie difficile. Tout d'abord, si les revenus sont plus élevés, les dépenses le sont aussi. A des paysans du district de Luoshan qui se plaignaient qu'un enfant du village gagne 70 yuan par mois comme ouvrier, on répondit en detaillant les dépenses auxquelles, avec sa femme, il devait faire face chaque mois : 19,5 yuan pour le grain, 2,4 pour les légumes, 1,5 pour le loyer, 2.8 pour l'électricité et 7,5 pour le chauffage, ce qui ne lui laissait que 14,4 yuan d'économies. Nul doute que ces dépenses fussent exagérées puisqu'un autre ouvrier devait affirmer. également pour les besoins de la cause, qu'avec 47 yuan par mois il pouvait parfaitement soutenir une famille de cinq personnes ${ }^{33}$. Mais les plaintes enregistrées par la presse au printemps 1957 donnent à croire que les citadins éprouvaient souvent de grandes difficultés à joindre les deux bouts.

A cela s'ajoutaient deux grands problèmes universellement présents : l'approvisionnement et le logement. On disait que "la pénurie de biens, les queues à tous les coins et la cherté de la vie sont la caractéristique des marchés de Zhengzhou $"{ }^{34}$. En effet, les rations céréalières étaient souvent insuffisantes, et il fallait souvent les compléter par des achats coûteux au marché noir. Pour recevoir ses tickets ou se procurer son grain, on devait parfois faire des queues d'une journée entière. Au reste, le comité de rue veillait à ce que des "économies" fussent réalisées et les voisins se montraient souvent prompts à dénoncer toute bombance anormale. Les plus graves difficultés d'approvisionnement concernaient les autres produits alimentaires, de plus en plus demandés depuis que le niveau de vie s'était amélioré, et notamment depuis la réforme des salaires de $1956^{35}$ : 
l'offre ne suivait pas la demande. Des réclamations particulièrement fréquentes portaient sur l'approvisionnement en viande. En effet, malgré une hausse des prix d'achat du porc et du bœuf, les arrivages de viande sur le marché officiel avaient été réduits par le mauvais état du cheptel et le développement du marché noir. Ainsi, l'approvisionnement de Zhenzhou avait baissé de $21 \%$ entre août et octobre 1956. De même, les légumes étaient rares sur le marché officiel, mais abondants sur les marchés libres, où ils subissaient des fluctuations de prix atteignant parfois $100 \%$. Il était tout aussi difficile de se procurer de nombreux biens manufacturés comme les vêtements, cela malgré certaines hausses de prix d'ailleurs mal accueillies par la population. On remarquera cependant que ces difficultés d'approvisionnement, surtout graves dans les villes nouvelles comme Hebi, Jiaozuo. Pingdingshan ou Sanmen et dans les quartiers neufs des grandes villes dont linfrastructure commerciale était insuffisante, sont rarement citées par nos sources pour les grosses bourgades rurales (Xuchang, Xinyang ou Nanyang). Le deuxième problème de la vie en ville. ceétait le logement ${ }^{36}$. En effet, la rapide croissance de la population citadine (qui avait plus que triplé depuis 1949) avait fait croitre la demande, et l'amélioration du niveau de vie avait fait apparaitre des exigences de qualité. Les locations de particulier à particulier étaient très onéreuses: jusquà 8 yuan par pièce dans le centre des grandes villes. La cherté des loyers et l'insuffisance des logements contraignaient de nombreux ouvriers à se serrer dans des galetas. A l'époque des grandes pluies de juillet 1956, une enquête officielle révéla que 58 des 197 logements occupés par les ouvriers de l'usine d'insecticides de Zhengzhou prenaient l'eau. Une préoccupation universelle était de se faire attribuer un logement dans les nouveaux quartiers mais les listes dattente étaient longues. Au début de septembre 1956, mille ouvriers de la construction de base de Zhengzhou se trouvaient sans logement déterminé. D'ordinaire, les entreprises modernes ne pouvaient. au bout de quelques années d'existence, loger qu environ la moitié de leurs employés et parmi eux. en priorité les apprentis et les célibataires. Aussi connait-on des cas de souslocation et même d'occupation sauvage ${ }^{37}$.

$\mathrm{Au}$ reste, s'ils étaient mieux aérés, plus propres et plus confortables (encore qu'ils ne disposassent pas tous de l'eau courante), les logements modernes ne présentaient pas que des avantages. Ils paraissaient assez chers à la location (au moins 1 yuan par pièce) et entraînaient de lourdes dépenses en eau et en électricité. Construits sur des plans soviétiques, ils se révélaient beaucoup trop chauds en été. Surtout, ils étaient trop étroits. Le règlement n'autorisait à délivrer d’appartement de deux pièces qu'aux familles de plus de six personnes. Les célibataires vivaient en général à plusieurs dans une même chambrée et, si d'aventure ils se mariaient, ils devaient attendre plusieurs années avant de recevoir un logement indẹpendant. Au total, donc, malgré dincontestables progrès, le logement restait l'un des points noirs de la vie urbaine. En juin 1957, une enquête officielle portant sur 201 familles de Zhengzhou révéla que la surface occupée par personne ne dépassait pas $4.39 \mathrm{~m}^{2}$, que 72 familles vivaient sans électricité et 135 sans eau courante. Comment ces conditions de vie n'auraient-elles pas entrainé des conséquences sociales néfastes ? On sent parfois, chez certains responsables, percer une inquiétude sur le développement de la criminalité chez les petits enfants du siècle des Sarcelles chinois. A Luoyang, le nombre des crimes et des infractions diverses a augmenté de $31 \%$ entre le premier trimestre de 1956 et le trimestre de $1957^{38}$. Agressions, petits larcins, disputes familiales... Le tableau est classique, et il vaut aussi pour les villes du Henan. On aimerait cependant disposer des informations nécessaires pour le préciser ${ }^{39}$.

\section{Une société de castes}

La vie. cependant, n’était pas également pénible pour tous. Il y avait un monde entre tel haut dirigeant qui, comme Pan Fusheng. réclamait un magnifique logement de fonction 
en se plaignant qu" " au Henan les manières soient campagnardes ", que "Zhengzhou soit si pouilleux qu'on ne peut pas y recevoir des hôtes étrangers $"{ }^{40}$, et l'ouvrier temporaire chassé de son village par les calamités naturelles. Mais il existait une différence encore plus significative entre tel chef de gare qui occupait indûment un deux-pièces et l'avait meublé grâce à des emprunts à son bureau ${ }^{41}$, et telle famille ouvrière de dix personnes. grands parents compris, qui s'entassaient dans une seule pièce. La différence des conditions était aisément perceptible dans la rue : il y avait les seigneurs, ceux qui roulaient à bicyclette ou plus rarement en automobile, et le petit peuple des piètons. Entre les deux, la différence ne tenait pas d'abord au revenu. Un " capitaliste national " pouvait très bien gagner plus de 200 yuan par mois et pourtant éprouver les plus grandes difficultés à joindre les deux bouts, ne serait-ce quà cause de ses charges fiscales. A l'inverse, un cadre rémunéré aux alentours de 70 yuan par mois se trouvait, grâce à ses multiples avantages de fonction, en situation bien plus favorable. Dans cette société à la fois mobilisée et dominée, ce neétaient pas les avantages matériels qui comptaient le plus, mais la situation de chacun par rapport au pouvoir. Que pouvaient bien peser le prestige passé. les villas et les concubines de certains "personnages démocratiques" au regard diune décision gouvernementale ou mème de la mauvaise humeur d'un petit cadre ? Avec son deux-pièces. notre chef de gare n’ètait quà peine mieux logé que ses ingénieurs - mais ceux-ci dépendaient de lui. Dans une société où tout avait déjà changé et où tout risquait de changer à nouveau, la différence essentielle séparait ceux qui changeaient et "rectifiaient les hommes" (zhengren) de ceux qui étaient seulement la main-d'œuvre anonyme des changements et la cible des rectifications. C'est pourquoi, à notre sens, trois grandes catégories sociales peuvent être distinguées dans la société urbaine du Henan en 1956-1957: celle des seigneurs, les cadres, qui étaient les maitres du changement et qui en profitaient le plus ; celle des ouvriers et, à un moindre titre, de la jeunesse scolaire qui en étaient en principe les bénéficiaires mais en fait l'outil ; et enfin celle de tous les autres, de tous ceux qui. à un titre ou un autre et à des degrés très divers, se trouvaient exclus du pouvoir et à sa merci. Le terme de caste que nous avons avancé plus haut est provocateur et peut-être faux. Mais il présente le mérite dattirer l'attention sur la rigidité de la hierarchisation sociale. Celle-ci n'était pas l'effet de la plus ou moins grande vitalité des groupes sociaux, mais bien plutôt la projection sur le corps social d'une vérité absolue, l'idéologie du régime. Si un individu ou une catégorie sociale se trouvaient exclus, cétait toujours pour des raisons de principe. C'était également pour des raisons fondamentales que les cadres bénéficiaient de tant d'avantages - ils représentaient l'avant-garde porteuse des idées justes ... et que les ouvriers étaient à la fois bien traités et dominés - ils constituaient la base sociale naturelle du régime.

\section{Les cadres}

Les cadres occupaient le sommet de la hiérarchie. Leur situation était enviée. C'est pour devenir cadre (ganbu) et monter ensuite dans la hiérarchie du pouvoir que l'on postulait l'admission au Parti et que l'on fréquentait les écoles du soir. Si le régime voyait dans le cadre un entraineur dhommes, la population le considérait dabord comme un individu qui ne travaillait pas ou pas toujours de ses mains, comme un privilégié qui profite du labeur accompli par les autres. De fait, les principaux attributs du cadre étaient un bureau et un stylo. Dans le langage commun, la distinction nétait pas toujours nette entre "cadres" et " personnel non productif ». Souvent, d'ailleurs, la meilleure façon de devenir cadre était dentrer dans le personnel de gestion. Ceci contribue à expliquer le gonflement du personnel non productif dans les entreprises industrielles : $16.8 \% \mathrm{du}$ personnel en moyenne dans toutes les usines de Zhengzhou. mais $23 \%$ à l'usine de coton $\mathrm{n}^{\circ} 3$ et $26,3 \%$ à l'usine de machines textiles, $30 \%$ pour toutes les usines de Luoyang. La 
proportion des cadres devait être également importante. Ils formaient, par exemple, $12 \%$ du personnel à l'usine de coton $\mathrm{n}^{0} 3$ de Zhengzhou ${ }^{42}$.

Bien qu elle fût enviée et, de fait, très favorisée, la situation de cadre n'était pas dépourvue de difficultés. Elle impliquait tout d'abord une véritable surcharge d'obligations bureaucratiques. En effet, la prolifération des administrations contraignait les cadres (surtout les plus gradés ou les plus favorisés par le pouvoir) à occuper concurremment plusieurs postes. Ainsi, le chef du bureau administratif de la région de Kaifeng cumulait à lautomne 1956 quatorze fonctions différentes ${ }^{43}$. Les cadres de base occupaient en général deux fonctions au moins, l'une dans le Parti et l'autre dans l'administration. et se trouvaient en permanence occupés à réaliser des enquêtes, à remplir des questionnaires ou à assister à des réunions.

Deuxième difficulté. qu'il ne faut pas sous-évaluer : rester en permanence " dans la ligne " de façon à ne pas prêter le flanc à la critique et à ne pas risquer d'être démis. Lorsque la ligne était claire. il fallait la faire appliquer sans défaut. De là un sens rigoureux de l'autorité qui, dans certains cas, justifiait des brutalités et des sévices ${ }^{44}$. Mais ladite " ligne " (luxian) paraissait fréquemment obscure. De plus, elle subissait des modifications presque constantes. "Étudier ", c'est-à-dire lire de près les journaux et les discours des plus hauts dirigeants. devenait donc non seulement une obligation professionnelle mais aussi une nécessité vitale. Par crainte d'un faux-pas, les cadres agissaient et parlaient avec la plus extrême prudence dans un débat politique. On évitait les locutions trop claires et lon utilisait avec circonspection la conjonction danshi qui, dans le langage politique chinois, marque le moment où ce qui est important va être dit.

La concurrence féroce entre les cadres rendait cette prudence plus nécessaire encore. Pour la carrière d'un cadre, l'impopularité auprès des masses constituait un bien moindre danger que la dénonciation d'un rival, mème en période de libéralisation. Lorsqu il se sentait menacé. le cadre pouvait se montrer féroce. Les jeunes "activistes" (jiji fenzi) étaient encouragés officiellement à critiquer leurs supérieurs. Ils ne s'en privaient pas, car là se trouvait pour eux le seul moyen de se frayer un chemin dans l'appareil. Aussi, les cadres se méfiaient-ils de leurs jeunes concurrents et s'efforçaient-ils de freiner leur carrière. Ainsi s'explique la longueur du stage probatoire des membres du Parti aspirants et plus généralement la faible ampleur du recrutement dans certaines entreprises ou localités pourtant politisées ${ }^{45}$.

Le cadre devait également se méfier de ses collègues. Ainsi, les frictions étaient fréquentes à la ville et à la campagne, entre les cadres locaux et les démobilisés de plus ou moins fraiche date ${ }^{46}$. Comme le reste de la population, les cadres de base jalousaient l'aide multiforme que recevaient les anciens soldats: à tous les niveaux, des "comités d'installation des démobilisés " étaient chargés de leur fournir un emploi. un logement et même, si nécessaire. une épouse ${ }^{47}$. Pour des raisons de principe, mais aussi parce que le pouvoir avait confiance dans l'intégrité et la discipline qui leur avaient été enseignées sous les drapeaux. les démobilisés se trouvaient systématiquement favorisés dans les embauches comme dans les promotions. Un grand nombre dentre eux étaient immédiatement recrutés comme cadres. Sur les 5100 anciens soldats et officiers installés pendant le premier semestre 1957 dans cinq districts de la région de Kaifeng, 3000 devinrent aussitôt cadres. De même. en ville, nombre de chefs de chantier ou de dirigeants d'usine étaient d'anciens officiers revenus à la vie civile à l'époque de la réforme agraire (tugai ganbu).

Or l'autoritarisme, dont les cadres d'origine militaire avaient fait preuve à maintes reprises. notamment lors du "premier bond" (1955-1956), avait laissé de mauvais souvenirs dans la population et suscité des rancœurs chez les autres cadres. A de nombreuses reprises. et malgré la propagande officielle, les cadres locaux et la population s'employèrent à gèner leur recrutement et à les forcer au départ. Étant l'objet d'une 


\section{LA CHINE CONTEMPORAINE}

suspicion générale et contraints de se frayer leur voie, les anciens militaires ne se gênaient pas de leur côté pour révéler les petits scandales locaux et imposer brutalement leur autorité. Au total, les résistances croissantes auxquelles ils se heurtèrent ne leur permirent, nous semble-t-il, que de coloniser des postes secondaires.

Les difficultés de la vie de cadre n'empêchaient pas, mais au contraire justifiaient officiellement les avantages dont jouissaient tous ceux qui disposaient d'une parcelle de pouvoir. Ces avantages étaient essentiellement des privilèges de fonction, difficilement quantifiables et très jalousés par la population ${ }^{48}$. Tout d'abord les cadres, et plus généralement les membres du Parti, étaient favorisés dans les nominations, les affectations, les promotions et les augmentations salariales. C'est ce que l'on avait bien vu pendant la réforme des salaires de 1956. La mensualité du chef du département... financier de l'université de Zhengzhou, était passée de 50 à 110 yuan entre le début de 1956 et le début de 1957. Diverses indemnités (notamment en cas de déplacement) s'ajoutaient au salaire de base. Et s'ils venaient à manquer d'argent, les cadres pouvaient toujours demander des prêts ou des secours (parfois plusieurs centaines de yuan) qui leur étaient accordés beaucoup plus libéralement qu'au tout-venant -- et qu'il leur arrivait bien souvent de ne pas rembourser.

De plus, tout cadre était considéré comme prioritaire pour l'attribution d'un logement neuf et pouvait s'arranger pour occuper plus de place que de droit. Le cadre équipait souvent son logis dappareils de luxe - par exemple, une radio - qui appartenaient à son bureau. A force dutiliser toujours la même bicyclette de fonction ou de se faire conduire dans la même automobile, il finissait par se les approprier. Un homme de sa condition pouvait rendre des services considérables à toute sa parentèle : à sa femme, procurer une place de cadre ou d'employée qui arrondirait les revenus du ménage ${ }^{49} ;$ à ses enfants, des places à l'école, à l'usine ou dans l'administration; à tous ceux qui l'approchaient, de précieuses " lettres de présentation " qu'il pouvait monnayer discrètement.

Mais les avantages matériels étaient sans doute moins importants que le prestige et le plaisir de dominer. Au théâtre, on attendait que les personnalités eussent rejoint leurs sièges réservés pour commencer la représentation. A la cantine de l'usine, les cadres disposaient généralement d'une salle spéciale où, contrairement aux ouvriers astreints à des services fixes, ils trouvaient à toute heure un bon repas chaud. Chez le coiffeur, chez le blanchisseur, dans tous les magasins, les cadres bénéficiaient de la priorité. A Luoyang, l'épouse d'un responsable municipal qui était venue pour avorter à l'hôpital fit chasser une sage-femme qui avait réservé le meilleur lit à une parturiente. Maître de l'interprétation et de l'application des lois, le cadre qui avait fauté bénéficiait des circonstances atténuantes à moins qu'il n'ait commis une erreur de type politique. Dans une usine de Luoyang, pour la même faute (des relations sexuelles en dehors du mariage), un ouvrier fut puni de deux ans de prison et un membre du Parti d'une simple rétrogradation de deux échelons. Tous les cadres dirigeants, certes, ne ressemblaient pas à ce chef de secrétariat du comité du Parti du district de Changge, un nommé Kong, qui, pour son seul plaisir, prolongeait jusqu’à trois heures du matin les représentations de la troupe de theâtre locale. Ce qui est cependant le plus remarquable dans le tableau que nos sources - protestataires, mais aussi officielles - dressent des cadres, c'est son manque de nuances. Excepté certains petits cadres et quelques héros de dévouement, tous les autres sont présentés comme faisant partie d'une même aristocratie prétentieuse et autoritaire ${ }^{50}$.

Mais l'avantage principal du cadre, celui qui en définitive expliquait tous les autres, c'était sa participation au pouvoir. Celle-ci ne lui donnait pas seulement les moyens disciplinaires d'assurer sa domination sur ses subordonnés, elle le mettait à même de conserver sa place en lui fournissant les seules informations réellement utiles dans un régime à la fois centralisé et mobilisé : les informations politiques. Le cadre était seul à connaitre l'interprétation conjoncturelle de l'idéologie officielle et ses applications 
légales ${ }^{51}$. Les cadres de chaque niveau recevaient une documentation spécifique qui, surtout lorsqu elle était de nature économique, ne pouvait être communiquée au commun des mortels. A Zhengzhou, il existait une bibliothèque de 30000 volumes, distincte de la bibliothèque provinciale et spécialement destinée aux cadres. Certes, le cadre ne savait pas tout ce que voulait et faisait Pékin, loin de là, car linformation lui était soigneusement distillée selon son grade. C'est ce que montre l'exemple suivant. D'après un " démocrate" de Luoyang, le discours que Mao Tse-tung prononça le 27 février 1957 sur La juste solution des contradictions à l'intérieur du peuple (et qui ne devait être publié en juin 1957 quaprès avoir été soigneusement révisé) comprenait 20000 caractères dans sa version originale ; dans sa version destinée aux cadres de niveau provincial, 8 à 9000 caractères : pour ceux de Luoyang, 3 à 4000 ; et pour les cadres d'usine, quelques centaines. Même ces derniers, cependant, se trouvaient bien mieux informés que le vulgaire, qui navait droit qu'à la presse et aux discours officiels, ou aux bruits les plus invérifiables.

On assistait pourtant, depuis l'automne 1956, à la naissance d'un véritable malaise chez une partie au moins des cadres. Jusqualors de plus en plus forte à mesure que le régime paraissait remplir ses objectifs et le pays progresser vers le socialisme, leur position avait commencé à s'effriter. D'un côté, l'échec agricole de l'été 1956 avait atteint leur popularité. De lautre, le pouvoir central lui-même limitait leurs prérogatives, les contraignait à consulter leurs ouailles et, en cas de conflit, ne les soutenait plus systématiquement. Dans les usines, ils devaient désormais réunir des " assemblées de délégués des ouvriers " souvent houleuses ${ }^{52}$. Ils étaient moqués, parfois insultés. Durant la campagne de rectification, certains dentre eux se plaindront de ne plus avoir l'autorité nécessaire ${ }^{33}$. La prolifération du marché noir et des trafics de toutes sortes ne se comprend pas sans cet affaiblissement de l'encadrement dans les villes. Faute d'informations, il est impossible de définir l'ampleur du phénomène. Sa gravité est cependant attestée par le fait que, pendant la campagne de rectification, deux catégories de cadres, de rang parfois moyennement élevé, sympathiseront avec les protestataires: des responsables de la politique rurale et des dirigeants de la culture.

\section{Les " créateurs du monde nouveau"}

Située au-dessous de celle des cadres, une deuxième catégorie sociale favorisée était celle des travailleurs de l'industrie. Ils étaient, si l'on peut dire, les jambes du régime puisque, d'après la vulgate, c'est leur labeur qui, grâce à la juste direction du parti communiste, engendrait le progrès économique. Quoique bien payés et bien considérés, les ouvriers formaient cependant une catégorie sociale hétérogène, qui souffrait de sa subordination et, dans certains secteurs. commençait même à s'agiter.

L'importance aussi bien mythologique qu èconomique de la classe ouvrière en faisait un rouage central de la société urbaine. Le régime attendait d'elle un comportement de classe, c'est-à-dire un soutien politique et un engagement enthousiaste dans la production. En échange, il lui accordait un statut matériellement et politiquement favorisé. De fait, les ouvriers recevaient des salaires mensuels de 30 à 100 yuan et bénéficiaient, comme dans le reste de la Chine, davantages sociaux remarquables. Ladhésion au Parti leur était relativement aisée et la qualité de fils d'ouvriers valait à leurs enfants des facilités de tous ordres. D'une certaine façon, l'ouvrier était le seul citoyen de plein droit du nouveau régime.

La réalité était cependant moins brillante et plus compliquée ${ }^{54}$. Tout dabord. les ouvriers ne se sentaient pas tellement privilégiés. Au même titre que les autres citadins. ils acceptaient mal les insuffisances et les inégalités du progrès, surtout la cherté de la vie et les difficultés du logement. Ils jugeaient leurs salaires insuffisants et critiquaient les disparités salariales entre secteurs ${ }^{55}$ et entre postes quavaient aggravées la réforme des salaires de 


\section{LA CHINE CONTEMPORAINE}

1956. Loin d'être accompli dans la joie, le travail était ressenti comme pénible, voire dangereux. Après s'être multipliés pendant le premier bond, les accidents du travail avaient diminué mais non disparu, car les machines étaient vétustes ou mal connues et les règlements ignorés. Une querelle permanente opposait les ouvriers et leurs cadres à propos des normes de production. Les premiers refusaient d'améliorer à la fois la quantité et la qualité. Ils contraignaient leurs supérieurs à opérer un choix plus ou moins clair entre ces deux impératifs, cela malgré les objurgations de la propagande. Moins aisément intégrables dans les statistiques et les rapports d'activité, les objectifs de qualité faisaient en général les frais de ce choix, à moins que des primes spéciales pussent être offertes.

Nos sources communistes décrivent des ouvriers dans l'ensemble peu soigneux et revendicatifs ${ }^{56}$. Les véritables grèves semblent avoir été rares, mais on trouve des allusions à des go slow et surtout à une mauvaise atmosphère dans les entreprises. La moindre indisposition donnait alors prétexte à une demande de congé de maladie. Les règlements de discipline, généralement sévères, étaient rarement observés, surtout dans les usines qui comprenaient une forte proportion de jeunes ou qui se trouvaient particulièrement touchées par la récession et tournaient au ralenti : le jeu, les bagarres, les beuveries même, paraissent alors avoir été chose fréquente. Les syndicats officiels, parfois tournés en dérision et désertés, ne pouvaient remplir leur fonction disciplinaire ${ }^{57}$.

L'importance des problèmes sectoriels qui agitaient la "classe ouvrière henanaise" nous conduit à voir plutôt en elle un agrégat composite de milieux différents. Les ouvriers expérimentés transférés de Chine de l'Est, en majorité employés dans les usines textiles de Zhengzhou, formaient un groupe à part. Bien payés, ils se plaignaient cependant du mauvais accueil des Henanais, de la différence des mœurs et de l'éloignement de leur famille ${ }^{58}$. Le directeur et les ouvriers d'une usine d'Anyang devaient même, par des grèves et l'envoi de délégations à Pékin, exiger collectivement le retour de leur entreprise à Shanghai ${ }^{59}$. L'emploi dans le secteur des services n'était pas recherché car la profession était surtout féminine, les salaires étaient faibles (de 15 à 40 yuan) et le travail pénible ${ }^{60}$. Une autre catégorie très spéciale était constituée par les ouvriers de la construction de base, pour la plupart temporaires, dont $80 \%$ étaient d'origine rurale : mal payés, brutalisés par les contremaitres, méprisés par la population, la plupart d'entre eux aspiraient à changer de condition, soit en retournant au village une fois leur contrat expiré et passées les calamités, soit en obtenant leur régularisation. De ce sous-prolétariat, nous ne savons malheureusement que peu de choses ${ }^{61}$.

Il existait également de nets clivages parmi les ouvriers d'une même entreprise. La presse en reconnaissait alors un, car il s'adaptait bien à la vulgate : celui qui aurait séparé les $30 \%$ de vieux ouvriers, grandis dans le malheur de l'ancienne société et conscients des bienfaits de la nouvelle, des $70 \%$ de jeunes ouvriers pour lesquels ces bienfaits étaient autant de dûs, qui introduisaient "spontanément" dans lentreprise des "idées non prolétariennes ". Cette analyse ne parait pas complètement exacte. A plusieurs reprises, certes, nos sources montrent de vieux ouvriers agacés par le comportement de leurs cadets et, plus généralement, de la jeunesse scolaire. Mais cet agacement tient moins à des divergences politiques qu'à des désaccords de type moral. Ce que les vieux ouvriers admettent mal, c'est l'introduction, aussi prudente soit-elle, par le régime de nouvelles attitudes à l'égard de l'autorité, de la famille et de la procréation qui rompent avec la tradition. Nous ne disposons d'aucune donnée précise qui permette d'affirmer que les vieux ouvriers étaient plus politisés et même proportionnellement plus nombreux dans le Parti que leurs cadets. Nul doute que, dans l'affirmative, la presse aurait livré d'abondants détails. Si les vieux ouvriers se sont montrés plus disciplinés dans le travail, ils ne se sont pas abstenus de revendiquer à l'instar de leurs cadets.

Mais le secteur le plus sensible du monde ouvrier était celui des jeunes apprentis ${ }^{62}$. Traditionnellement sous-payés (moins de 30 yuan par mois) et brutalisés par les autres 
ouvriers, ils recevaient d'ordinaire leur titularisation au terme de contrats d'apprentissage très courts. Or, à cause de la récession, ceux-ci furent rallongés au début de 1957 par les autorités. Se sentant injustement traités dans une période où la tendance était plutôt au relâchement des contraintes, probablement influencés par les troubles scolaires et universitaires qui se produisaient alors, les apprentis n'hésitèrent pas à protester par tous les moyens, jusques et y compris la grève avec occupation des locaux. Ainsi, au printemps de 1957, les 379 apprentis de l'usine de tracteurs de Luoyang qui se trouvaient en formation à l'usine de machines textiles de Zhengzhou fondèrent un comité d'occupation dont le slogan était «non au compromis, non à la trahison", avant de devoir pour la plupart céder à l'autorité.

\section{Les " jeunes pousses"}

Le problème des apprentis était lié à celui, plus général, de la jeunesse scolarisée. Cette couche sociale, elle aussi très favorisée, posait au pouvoir un problème nouveau. Favorisés, les jeunes létaient évidemment par les possibilités scolaires et professionnelles que le régime leur ouvrait, ainsi que par l'attention qui était accordée à leur formation intellectuelle et politique : ils représentaient l'avenir. Mais, depuis l'été 1956, diverses circonstances avaient fait apparaitre les limites et mème les contre-effets des progrès accomplis. Tout d'abord, l'échec du premier bond avait entraîné des conséquences néfastes sur la situation scolaire ${ }^{63}$. Pour de nombreuses familles $(42 \%$ des parents délèves du lycée de Lankao), le poids des écolages n était plus supportable. Mal nourris et mal vêtus, les lycéens du Henan avaient passé un hiver difficile et fréquemment connu des problèmes de santé. Ainsi, dès l'automne 1956, la moitié des élèves d'une classe d'un lycée de Kaifeng avaient perdu du poids. Bien plus grave aux yeux d'une population traditionnellement disposée à consentir des sacrifices pour l'éducation de ses enfants, le ralentissement des constructions scolaires, provoqué par la récession économique, avait limité les possibilités d'accueil des lycées. Lors de la rentrée scolaire de l'automne 1956, ceux-ci n'offraient, dans toute la province, que 100000 places de secondaire aux 390000 diplômés de l'enseignement primaire. La rentrée de l'automne 1957 ne s'annonça guère mieux puisque la moitié seulement des diplômés du primaire purent entrer au lycée et le tiers des diplômés du " secondaire inférieur" (chuzhong) dans le "secondaire supérieur" (gaozhong) alors qu'à l'échelle de la province seulement 3000 des 9000 diplômés du "secondaire supérieur " trouvèrent place dans les universités et écoles spécialisées. Un plus grand nombre de diplômés se présentèrent donc sur le marché du travail au moment où les capacités d'absorption de l'industrie diminuaient. On tenta de résoudre le problème en encourageant la formation d' "écoles secondaires libres " et surtout, en envoyant ou renvoyant les diplômés du primaire à la campagne, mais d'abord sans grand succès. Le désœuvrement des jeunes posa dans les villes des problèmes d'ordre public sur lesquels nous sommes malheureusement trop mal renseignés.

Difficultés matérielles, compétition accrue dans les examens, incertitude sur les débouchés : tout cela explique largement la mauvaise atmosphère qui régnait dans les écoles secondaires ${ }^{64}$. Les écoliers protestaient contre le mauvais état des locaux et l'insuffisance de la nourriture. Après l'échec des coopératives et les événements de Hongrie, lagitation prit une tournure franchement politique dans quelques lycées au moins. A Miyang, on brocardait le mouvement des coopératives. Dans un lycée de Lankao, il fallut l'intervention du chef du district en personne pour faire cesser les revendications et interdire les slogans pro-hongrois. Au total, le comportement sociopolitique des lycéens henanais semble avoir été assez proche de celui de leurs aînés du supérieur qui devaient, au printemps 1957, annuler ou renverser le pouvoir du Parti dans 


\section{LA CHINE CONTEMPORAINE}

plusieurs universités. Bref, comme la classe ouvrière, les « jeunes pousses " ne se laissaient pas dresser aussi docilement que prévu.

\section{Les exclus}

En dessous ou plutôt à la périphérie des couches privilégiées, divers groupes sociaux peuvent être classés dans une même catégorie : celle des exclus. En effet, quoique leur revenu et même leur statut social fussent très différents, ces groupes sociaux se trouvaient dans une situation. quant au fond, comparable car ils navaient aucune part au pouvoir et en dépendaient pour leur survie.

Le premier cercle était celui de l'exclusion par la profession. Le degré de l'exclusion et du mépris subis par ces minorités professionnelles variait considérablement. La profession médicale, par exemple, représentait un cas limite. L'insuffisance des équipements (malgré les progrès accomplis), la longueur et la pénibilité du service provoquaient de multiples plaintes. La profession dans son ensemble se sentait injustement traitée : s'il développait la santé publique, le régime se préoccupait tout de même plus de la société que des individus et, si l'on peut dire, des âmes que des corps. Une autre catégorie de médecins, ceux qui soignent et transforment le corps social - les cadres - tenaient le haut du pavé. De plus, chaque branche de la médecine, chaque personnel médical avait ses propres motifs d'insatisfaction. Les praticiens de la médecine chinoise s'estimaient défavorisés par rapport aux spécialistes de la médecine occidentale, les autres se plaignaient de l'influence excessive réservée par le régime à la médecine soviétique. Dans les hôpitaux, on protestait contre les horaires trop lourds, la rémunération trop faible et surtout la pression du Parti. Un médecin déclarait: "Le jour il me faut soigner et la nuit mautocritiquer. " Les infirmiers déploraient leurs bas salaires, les logements calamiteux et surtout les brimades dont ils étaient l'objet de la part des cadres ${ }^{65}$.

Il faut toutefois remarquer que le revenu des médecins et des infirmiers était presque équivalent à celui des ingénieurs et des ouvriers : ce n était pas la misère, loin de là. De plus, la profession en tant que telle n'était pas menacée de disparition. Son mécontentement semble tout autant dú aux souvenirs de l'ancien régime et à la connaissance des avantages acquis par les médecins occidentaux qu à son exclusion effective de la société chinoise. Enfin, si ce mécontentement nous est connu, c'est parce que les médecins et les infirmiers disposaient des moyens intellectuels et matériels de lexprimer : en ce sens au moins, ils étaient bien plus favorisés que d'autres exclus.

Le cas des artisans, sans être dramatique, parait plus grave. Cette couche sociale réagissait mal aux transformations brutales qu elle avait subies en 1955-1956. Embrigadés dans des coopératives souvent trop grandes et mal gérées, conscients que les contraintes collectives se relâchaient un peu partout, les artisans ne mettaient guère de cœur à l'ouvrage. Chaque fois que cela était possible, ils quittaient leurs coopératives ${ }^{66}$. Une solution simple était encore plus répandue : se contenter de faire acte de présence dans la coopérative et organiser par ailleurs de véritables ateliers clandestins employant de la main-d'œuvre d'immigrants illégaux, souvent reliés entre eux et fournissant même des entreprises publiques. Dans le seul quartier du Sept février à Zhengzhou, on devait dénombrer, à l'automne 1957, 156 de ces "entreprises noires" ${ }^{67}$. Si les artisans étaient encore méprisés et brimés en 1956-1957, c’était pour des raisons idéologiques et psychologiques, non économiques, car depuis l'été 1956 on reconnaissait à nouveau l'importance des activités artisanales. Malgré ses tendances inévitablement "petitesbourgeoises ", un bon artisan conservait donc de bonnes chances de survivre. Ce nètait déjà plus le cas des petits marchands de tout acabit - 1700 à Zhengzhou dans l'été 1957 - qui animaient les foires rurales et, en ces temps de rareté, ravitaillaient les villes ${ }^{68}$. Quoique officieusement admis tant que la situation économique resterait médiocre et le 
prestige du Parti diminué, ils se trouvaient condamnés à terme comme catégorie professionnelle. Conscients de cette menace. et aussi des possibilités qui souvraient temporairement à eux. ils profitaient des derniers arpents de liberté que leur abandonnait le régime. Ainsi, deux marchands de Huaxian accumulèrent un bénéfice de 23800 yuan dans la première partie de 1957 en allant vendre au Gansu des matériaux de construction. Parmi les trafics les plus fréquemment cités, on note la vente au marché noir des légumes produits dans les banlieues, la revente des tickets de céréales et de coton et aussi la réparation des machines que certaines entreprises préféraient confier à des bricoleurs plutôt qu'aux ateliers officiels. D'autres faits divers donnent à croire qu'en réalité tout un chacun cherchait à "se débrouiller». C'est ce que montrent deux exemples pris aux extrêmes de la hiérarchie sociale : envoyé en mission officielle à Canton, un cadre y acheta huit montres à 80 yuan la pièce, qu'il revendit à son retour au Henan pour 125 yuan l'une; un évadé de camp de rééducation avait installé à Zhengzhou une officine de faux billets dont il écoula 580 yuan en se prétendant "cadre d'État ". La généralisation des trafics de toutes sortes est un aspect important de la vie urbaine en 1956-1957. Mais elle ne doit pas faire oublier la fragilité de la situation de tous les débrouillards : le régime les écrasera dès qu'il le jugera utile.

Du moins leurs crimes ne pourront-ils pas atteindre le plus haut niveau de gravité. Ce n'ètait pas le cas des minorités ethniques et religieuses qui peuplaient le deuxième cercle. Celles-ci étaient par définition suspectes des fautes les plus infâmantes. Peu nombreux ( $1 \%$ de la population provinciale) mais concentrés dans quelques villes où ils vivaient autrefois de petits commerces, les musulmans du Hénan avaient souffert de la brutalité du mouvement de socialisation et se plaignaient du mépris du Parti pour leurs coutumes ${ }^{69}$. Du moins, le pouvoir admettait-il la nécessité de les ménager.

Il en allait autrement, en fait, des quelques chrétiens, dont le clergé, même s'il avait dénoncé tout lien avec l'Occident, ne pouvait exercer. Aussi une minorité résolue, connaissant son destin, s'opposait-elle à la fois à l'Église officielle et au régime ${ }^{70}$. Le dernier cercle, celui des exclus politiques - environ $0,5 \%$ de la population ${ }^{71}$ - doit-être considéré comme l'antichambre de la mort. A force de bons comportements, certains anciens capitalistes liés au Kuomintang et officiers de l'armée nationale avaient acquis le droit de survivre. Mais les autres, qu'ils fussent ou non placés sous surveillance, croupissaient dans la misere et mouraient parfois de mauvais traitements ${ }^{72}$. Ils composaient une bonne partie de ces "foyers en difficulté " que les calamités de 1956 avaient souvent précipités dans la disette. En ville, certains d'entre eux tentaient d'éviter la misère en exerçant de petits métiers. Mais ils vivaient sous la menace permanente d'une campagne répressive ou d'une fausse accusation.

En général, une partie de leur famille avait été exécutée ou vivait dans des camps de rééducation. Nos sources ne font malheureusement que de rares allusions à l'existence de camps au Henan ${ }^{73}$. Nous connaissons l'existence, à l'intérieur du Bureau provincial de la sécurité publique, d'un Bureau d'administration de la rééducation (laogai guanli ju). De ce Bureau dépendaient au moins une briquetterie à Pingdingshan et des travailleurs de l'énorme ferme d'État de Minquan. On peut émettre aussi l'hypothèse que l'agitation sociale de l’époque n èpargna pas les camps, car deux évasions et même des troubles sont attestés.

\section{Le troisieme monde}

Entre les exclus et les privilégiés subsistait, à l'époque, un troisième monde au destin incertain. Il était composé des milieux sociaux " démocratiques " que le régime prétendait toujours rallier : la bourgeoisie nationale et les intellectuels formés sous l'ancien régime. Dans ce troisième monde, les conditions matérielles étaient très diverses. Certains 


\section{LA CHINE CONTEMPORAINE}

"personnages démocratiques » conservaient encore un grand train de vie. Les anciens propriétaires d'usines grandes et moyennes touchaient des revenus confortables, encore qu'ils ne fussent pas à l'abri des rappels d'impôts et qu'ils ne disposassent d'aucune protection sociale. Ingénieurs et professeurs recevaient des salaires confortables. Mais il existait aussi, surtout dans les grosses bourgades rurales, toute une frange de petits hommes d'affaires ruinés par les impôts, de professeurs à la retraite ou de petits employés aux écritures qui échappaient difficilement à la misère. On citait par exemple le cas de la veuve d'un de ces petits hommes d'affaires qui, pour survivre, avait dû épouser -humiliation suprême $\cdots$ un cuisinier ${ }^{74}$. Ceux-là se plaignaient de ne pas être assez défendus par les partis démocratiques qui les représentaient en principe et ils ne souhaitaient qu'une seule chose : changer de statut et être amalgamés à la classe ouvrière afin de bénéficier de la sécurité de l'emploi et d'avantages sociaux.

Lunité de cette couche sociale dérivait non seulement de son passé privilégié, mais aussi de son statut présent et de son avenir incertain. Quoique entourés de prévenances, parfois installés à des postes de pouvoir, ses membres étaient l'objet d'une surveillance de tous les instants et avaient toujours subi les décisions du régime. L'opinion publique ne s'y trompait pas: même quand ils occupaient des fonctions prestigieuses (ingénieur, professeur), leur réputation était médiocre. Cependant, depuis l'été 1956, le régime avait réévalué leur statut, tout en limitant le pouvoir des cadres. Avant même qu'ils fussent publiquement conviés à donner leur avis au printemps 1957, les démocrates ne cachaient pas leurs aspirations : alors que les optimistes et les plus nantis espéraient un renforcement de leur statut social et de leur rôle politique, les autres, au contraire, habitués à craindre tout changement, ne voyaient de salut que dans leur déclassement et dans un impossible amalgame à la classe ouvrière. En tout cas, chacun d'entre eux espérait une clarification de son statut. Celle-ci devait survenir brutalement dans l'été 1957: ce troisième monde disparut corps et biens.

\section{Une société froide}

On aimerait décrire par le détail la vie quotidienne à Zhengzhou ou Luoyang en 19561957. Nos sources ne le permettent pas. Elles laissent toutefois une impression d'ensemble assez nette : à peine sortie du conformisme et des drames de l'avant-guerre, la nouvelle société s'est donnée de nouveaux maitres d'une rigueur extrême. Cette société froide rendait pratiquement impossible des relations humaines autres que politiques, professionnelles ou familiales. On n'osait pas réunir une petite fête de peur d'une dénonciation. Les vieux amis ne s'invitaient plus pour ne pas se voir reprocher des «liens factionnels " ou, s'ils ne parlaient que de choses banales, des "sentiments apolitiques". Quand ils ne pouvaient pas séviter, ils commentaient le temps qu'il faisait... A la cantine de l'usine, chacun mangeait en silence ${ }^{75}$. De plus, après avoir détruit la grande famille traditionnelle, le nouveau régime avait en partie réhabilité un moralisme qui venait de loin, ce qui n'allait pas sans contredire sa propagande sur l'émancipation de la femme. Les enfants étaient fermement rappelés au respect de leurs parents. Les époux querelleurs n'obtenaient le divorce qu'en cas de force majeure ${ }^{76}$. A l'usine, les amitiés entre garçons et filles suscitaient la méfiance ou des plaisanteries graveleuses. Ce conformisme moral favorisait le maintien de certains privilèges traditionnels du mâle. Dans les grands magasins, des clients serraient de près les jeunes employées. Il arrivait que les cadres ou même des professeurs profitassent de leur statut pour abuser de leurs subordonnées ou de leurs élèves ${ }^{77}$.

Il ne faut cependant pas noircir excessivement le tableau. Tout d'abord, le régime n'avait pas sérieusement tenté de contrôler la vie familiale. Cet immense domaine du vécu social lui échappait encore complètement. C'est ce que montrent aussi bien son incapacité 
à persuader les familles de ne pas trop pousser leurs enfants vers les études que la remarquable discrétion de la campagne de limitation des naissances dans la province. De plus, par sa lourdeur, la pression idéologique du régime produisait des contre-effets. Les défoulements populaires n'ètaient pas toujours innocents. Les " livres jaunes ", c'est-à-dire les romans érotiques, faisaient toujours des ravages dans les lycées ${ }^{78}$. Il se produisait parfois de violents désordres dans la rue ainsi que dans les arènes sportives ou dans les salles de théatre. Est-ce seulement à cause de l'amour traditionnel des Henanais pour le theatre ou aussi parce que lindiscipline pouvait prendre un tour chauvin, voire contestataire? La presse provinciale s’est fréquemment plainte des chahuts déclenchés par les spectateurs lors de la venue de troupes dautres villes, dautres provinces et même étrangères ${ }^{79}$. La nuit, dans les grandes villes, les abords des quartiers neufs neetaient pas toujours sûrs. Bref. canalisée ou réprimée dans la vie officielle et dans la vie diurne. la violence se réfugiait dans cette partie de la vie sociale que le régime ne pouvait ou ne voulait pas contrôler totalement : le sport, le spectacle, la vie nocturne, la famille peut-être. On souhaiterait disposer dinformations plus nombreuses encore sur cette partie non contrôlée de la vie sociale afin de préciser une hypothèse qui mérite en tout cas d'être formulee : l'agitation politique des Cent-Fleurs serait partiellement l'effet de la répression morale et sociale sévère subie par la société urbaine. L'excès même de la " froideur " aurait été une cause de l'embrasement du printemps 1957.

\section{Qu'est-ce qu'une crise sociale en Chine populaire?}

Cette longue description achevée, revenons maintenant à notre interrogation initiale. Fallait-il, faut-il encore parler de crise sociale au Henan en 1956-1957? Pour une fois. notre inquiétude ne provient pas seulement de l'état des sources. En effet, même si celles-ci demeurent insuffisantes et inégales, elles sont en l'occurrence plus abondantes, plus concrètes et plus franches que d'ordinaire. A partir de l'automne 1956, la presse a publié plus de « matériaux négatifs " quauparavant et les prises de parole du printemps 1957 ont mis au jour des réalités soigneusement cachées jusqu'alors. Les matériaux datant de cette époque sont très probablement, avec ceux de la Révolution culturelle, les plus riches dont nous disposions sur la société chinoise depuis 1949.

Il s’agit, en fait, d'un problème de définition. Le terme de crise indique couramment. soit une rupture par rapport à la normalité. soit l'aggravation considérable et rapide d'une maladie. Une crise sociale affecte une importante partie de la population. Celle-ci prend conscience du fait que sa situation matérielle se dégrade. en rend responsable le pouvoir politique et cherche à échapper à ses lois en protestant et en brisant concrètement la discipline : une crise sociale se traduit par des manifestations de mécontentement plus ou moins violentes, par des troubles. Si la population ne donne pas à son mécontentement une expression politique directe qui viserait à remplacer le pouvoir, elle produit toutefois dans un délai plus ou moins proche des conséquences politiques de nature variable.

Or. cette définition de la crise sociale ne s'adapte que partiellement au cas de la société henanaise en 1956-1957. Il est vrai quà la campagne l'échec agricole du premier bond a provoqué une détérioration des relations entre les masses paysannes et le pouvoir, détérioration dont le signe le plus visible fut l'extension des phénomènes d'indiscipline économique. De même, dans certaines couches de la population urbaine comme les apprentis et la jeunesse scolarisée, on note un début dagitation, alors que par ailleurs le mécontentement parait général. Cependant, il faut bien reconnaitre que l'aggravation de la situation sociale na pas été, surtout dans les campagnes. à la mesure de la dégradation de la situation économique. Les terribles souffrances infligées à la paysannerie par une campagne d’intensification de la production puis par de très dures calamités naturelles 


\section{LA CHINE CONTEMPORAINE}

n'ont provoqué que de nombreuses désobéissances, mais aucune révolte, aucun mouvement d'opposition violent et de grande ampleur. La masse s'est surtout employée à limiter les conséquences matérielles des calamités en profitant des libertés qui lui étaient temporairement rendues. A la ville, une étroite minorité s'agitait, mais elle ne disposait d'aucun poids économique et politique ; la majorité était mécontente et résignée. De plus, aucun lien de cause à effet n'a existé entre la situation sociale en Chine depuis l'été 1956 et le déclenchement de la campagne de rectification du printemps 1957, qui releva dune décision politique. L'existence de la crise sociale a seulement pu être l'un des motifs (important, certes) qui ont poussé les dirigeants centraux à déclencher cette campagne et surtout, quand elle a menacé de dégénérer, à l'interrompre. Tout se passe comme si, à cette époque, les phénomènes sociaux ne jouaient qu un rôle causal faible (ou bien médiatisé par les dirigeants) dans l'évolution politique. En ce sens aussi, le terme de crise sociale paraît abusif.

Pour expliquer cet état de choses, il est toujours possible d'évoquer des facteurs d'ordre psychologique : des habitudes séculaires de passivité et de scepticisme à l'égard de tout pouvoir, le souvenir des horreurs d'avant 1949, peut-être aussi, chez certains, un réel espoir dans l'aptitude d'un régime encore jeune à se corriger, à quoi il faudrait ajouter le sens proprement henanais de la discipline. Mais il parait plus sûr de rechercher une explication dans la nature même du régime politique fondé en 1949 à léchelle de toute la Chine. Par définition, il sagit d’un régime dictatorial, une "dictature du prolétariat" exercé par son expression politique, le parti communiste. Par nature, ce régime se montre arbitraire et policier. Il soulève inévitablement des sentiments de rancœur et d'hostilité dans la population. Cependant. malgré ses imperfections, le contrôle du Parti sur la population est ordinairement assez puissant pour en interdire l'expression ouverte. $A$ fortiori, une agitation populaire de grande ampleur et une crise sociale ouverte au sens où nous l'entendons en Occident sont matériellement impossibles et même inimaginables en Chine populaire. Que le parti communiste se trompe, que même il devienne sanguinaire, et les signes de protestation resteront extrêmement ténus sur le moment. La domination du Parti sur la population et linvestissement du social par le politique expliquent qu'en Chine des erreurs, qui dans tout autre régime auraient provoqué une crise de grande envergure, n'ont entrainé que de faibles conséquences sociales et, dans l'immédiat, aucun changement politique majeur.

En fait, la société chinoise est à ce point dominée que toute réaction sociale d'une certaine ampleur à la politique du pouvoir ne peut prendre naissance et se développer qu'avec une véritable autorisation de tout ou partie de la direction centrale. La société ne peut réagir de façon sensible que si elle y est autorisée ou invitée. La permission peut être plus ou moins franche et libérale : de là vient la différence entre des événements comme la crise de 1956-1957, la Révolution culturelle et mème les troubles de la place Tiananmen en avril 1976 ou la contestation de l'hiver 1978-1979. Dans le cas de la société henanaise en 1956-1957, les réactions populaires que nous avons notées n'auraient pu s'exprimer sans l'assouplissement de la politique sociale et le début de libéralisation politique décidés au préalable par Pékin.

Cependant, les masses ne peuvent jamais être complètement manipulées. Et, où que ce soit, tempérer une tyrannie est non seulement difficile, mais dangereux. Difficile : encore sous l'influence des glaciations précédentes (et aussi dotee par l'expérience d'un solide bon sens), la population ne se convainc que très lentement de la réalité et de la durabilité du changement ; en attendant des preuves décisives, elle se contente, dans sa majorité, de profiter au jour le jour des avantages qui lui sont concédés. Dangereux : la minorité de ceux qui n'ont rien à perdre peut profiter de l'occasion pour bousculer les règlements et exprimer une critique radicale ; n'assignant plus de limites à son action, mais incapable de lui donner un but positif, elle ne peut se livrer qu'à une action désordonnée. A l'apathie de 
la masse correspond l'agitation divagante des minorités. C'est en ce sens, et en ce sens seulement, qu'il nous parait possible de parler de crise sociale au Henan en 1956-1957. Étant donné la nature dictatoriale du régime communiste chinois, ce que la société henanaise a manifesté pendant ces années représentait le maximum admissible par le pouvoir. On retrouve alors les principaux traits communs à toutes les autres crises sociales survenues en Chine populaire : la faible ampleur des incidents, leur manque partiel de spontanéité et un net contraste entre lapathie de la masse et l'agitation des minorités. Sous cet angle ${ }^{80}$, les analogies sont évidentes avec la période des « années noires " (1960-1962) et même avec certains aspects de la Révolution culturelle.

Ces éclaircissements permettent de poser plus clairement le problème trop rarement abordé des rapports entre la crise sociale de 1956-1957 et la crise politique du printemps $1957^{81}$. Car si la crise sociale n’a pas exercé d’influence directe sur le cours des événements, ses effets indirects ont été d'une importance considérable.

En effet, elle a contribué à renforcer les divisions à l’intérieur du Parti. La tyrannie maoiste marche sur la tête. L'autorité politique a mille fois plus de poids que le corps social. Seule la demi-fiction de la ligne de masse garantit la possibilité dun aller-retour du politique au social. Dans les faits, laller est beaucoup plus rapide et plus important que le retour. Le régime est beaucoup plus disposé à parler quà écouter : c'est pourquoi il entend mal. De son côté, la population ne peut évidemment risquer de dire la vérité, mais elle ne peut non plus éviter de dire quelque chose quand on lui demande son avis, et par là de révéler plus ou moins clairement ses sentiments. La crise sociale de 1956-1957, c'est l'expression nécessairement prudente du mécontentement maximum. Il y a donc dialogue (si l'on peut dire) entre des sourds (les dirigeants centraux) et des muets (le peuple). En lançant le mouvement de rectification davril 1957. c'est-à-dire en faisant fond sur ladhésion de la population aux objectifs du parti communiste, Mao Tse-tung a manifesté une incompréhension totale des aspirations populaires. Sa démarche était en l'occurrence dictée au moins autant par la surdité que par l'idéalisme.

Mais dans ce régime il en est qui, par situation et pour ainsi dire par profession, sont moins sourds que les autres : les cadres moyens et inférieurs qui composent l'immense majorité de l'appareil du Parti. Entre les deux termes de l'alternative du printemps 1957 donner au mouvement de contestation le temps de se définir ou refermer précipitamment les portes qui venaient de lui être ouvertes - leur réponse était toute prête et presque automatique. Sans avoir tout compris, ils en avaient assez entendu, ils en savaient assez pour prévoir que la contestation menacerait dabord leur propre pouvoir et bientôt le régime lui-même. De là des tiédeurs, des maladresses plus ou moins voulues et des obstructions caractérisées qui joueront leur rôle au printemps 1957. De là aussi des divisions, au niveau provincial comme au niveau central, entre les dirigeants plus sensibles à l'utopie maoiste (ou aux ordres du Comité central) et ceux dont le comportement était d'abord dicté par ce que l'on pourrait appeler des intérêts de classe. Ces derniers étaient sans doute les plus raisonnables, tant du point de vue de leurs propres intérêts que de la stabilité du régime. En effet, la crise sociale fournissait aux contestataires des thèmes d'intervention publique explosifs et un public tout prêt à les approuver. Les orateurs du printemps 1957 ne feront souvent que répéter tout haut ce qui se chuchotait tout bas et rapporter ce qu'eux-mêmes avaient constaté ou vécu. Tout cela. en des temps de rareté et de mécontentement, n'était, ne pouvait pas être académique. Derrière les déclarations encore prudentes des libéraux, tout un mécontentement populaire menaçait de se faire jour, cette fois-ci franchement et violemment. De tous ces pantins bavards, la crise sociale menaçait de faire des révolutionnaires. Elle leur offrait le redoutable avantage de pouvoir être entendus par la masse. Par là. le régime étant ce qu ïl était, elle les condamnait à une fin rapide.

Avançons donc une conclusion provisoire : leetroitesse de la domination exercée par le 


\title{
LA CHINE CONTEMPORAINE
}

pouvoir politique sur la société explique qu'en Chine maoïste la crise sociale ne revête qu'une ampleur sans commune mesure avec la gravité des maux qui l'ont provoquée ; elle explique aussi que. bien loin d'influer directement sur le pouvoir, celle-ci dépende de son assouplissement pour se manifester; le développement de la crise sociale modifie cependant de façon indirecte le débat politique en aggravant son enjeu et en favorisant lintensification des divisions à l'intérieur du Parti. Provisoire, cette conclusion risque aussi de se révéler imprudente si de nouvelles monographies sont réalisées, qui porteront sur d'autres crises traversées par la société chinoise (notamment la plus grave, celle qui a suivi l'échec du Grand Bond en avant) et qui mettront à profit des sources plus variées, plus nombreuses et aussi plus vraies.

\author{
Jean-Luc DOMENaCH \\ Fondation nationale des sciences politiques \\ (CERI)
}

\section{NOTES}

1. Ce texte est extrait, dans une version légèrement modifiée et raccourcie, d'une étude plus vaste, Aux origines $d u$ Grand bond en avant: la crise économique, sociale et politique d'une province chinoise en 1956-1957, à paraitre en 1980 aux Presses de la Fondation nationale des sciences politiques.

2. A savoir principalement le Quotidien du Henan (Henan ribao, ci-après HNRB), le Quotidien de Zhengzhou (Zhengzhou ribao, ci-après ZZRB) et le Quotidien de Luoyang (Luoyang ribao; ciaprès $L Y R B$ ).

3. $H N R B, 4$ juil. 1958.

4. Zhongguo nongbao (Journal de l'agriculture chinoise), 8 août 1957, pp. 13-14; Zhengzhou shifan xueyuan dilixi (Département de géographie de l'École normale de Zhengzhou) : Henan dili (Géographie du Henan). Editions populaires du Henan, 1959, p. 82.

5. Voir dans $H N R B, 10$ nov. 1956, lexemple precis du district de Xiuwu.

6. Sur les calamités naturelles du Henan en 1956. qui touchèrent plus de la moitié des districts, voir surtout $H N R B, 20$ août et 23 nov. 1956.

7. A propos de la situation des paysans des zones sinistrées, on consultera HNRB, 25 déc. 1956. 17. 24 mai, 11. 26 juin, 7, 14.24 juil., 14 août 1957 et Renmin ribao (Quotidien du peuple, ci-après $R M R B), 21$ mai 1957.

8. HNRB, 7 juil., 2, 4, 5, 8 sept., 27 oct. 1956, LYRB, 27 mai. 7 juin. ZZRB, 24 juin 1957.

9. $H N R B, 14$ juil., 9, 16 aoüt, 3, 8 sept.. 23 oct.. 2 nov., 1956. 4 janv., 5 juin 1957.

10. Cet état desprit est attesté notamment dans $H N R B, 10$ janv., RMRB, 26 mars, $L Y R B, 23$. 28 mai 1957.

11. Dagongbao (L'impartial, ci-après $D G B), 8$ août 1957.

12. Sur les retraits des coopératives, voir notamment $H N R B, 22$ juin, 14 juil., 3, 13,18 août 1957, 20 janv., 4, 17, 18, 20, 25. 29 juil.. 4, 7. 11, 18 août 1958 et $R M R B, 10$ mars 1957.

13. Les campagnes du Henan abritaient plus de huit millions de familles. Ezra Vogel fournit également le chiffre assez faible de 130000 départs temporaires et de 20000 définitifs dans la province du Guangdong (Canton under communism, New York. Harper, 1971, p. 204).

14. $H N R B, 9,10,15$ août. 3 sept. 1957.

15. $L Y R B, 28$ mai, $H N R B, 15,29$ juil., 2. 3 août, $D G B, 5,8$ août, $H N R B, 10,11,15,18,28$, 
29 août. $Z Z R B,\left.\right|^{\text {er }}$ sept.. $H N R B, 8,15,20,25.26,29$ sept. $4.7,18$ oct. 28 déc. 1957, 11 août 1958.

16. Mais la surface effectivement récupérée a été étroite dans le seul cas précisément connu : un peu plus dun hectare pour tout le district de Minquan (HNRB, 11 aoút 1958).

17. Comme ce fut le cas dans une cooperative de la banlieue de Zhengzhou où les "représentants des paysans" furent brutalement persuadés par les cadres de demander la... suppression des lopins de terre privés ( $H N R B, 8$ juin 1957).

18. Les 473 membres d'une coopérative de Changge avaient été mis à l'amende en juillet 1956 : sur les 96 cadres de base d'une coopérative de Guangsha. 15 distribuaient couramment des injures et 10 des coups ; dans une coopérative de Lingbao, 1131 journées de travail avaient été dépensées en indemnités de réunions dans une année : les cadres remboursaient rarement leurs emprunts. Cf. $R M R B, 25$ avril, $L Y R B, 20,28$ juin, $H N R B, 3,10$ nov., 8 déc. 1957.

19. Voir par exemple $L Y R B, 23,28$ mai. $H N R B, 2$ juin. 8,10 août, $15,17,28$ sept., $Z Z R B$, 10 oct. . $H N R B, 16$ oct.. 30 nov. 8 déc. 1957.

20. $D G B, 8$ août 1957.

21. HNRB. 15.17 et 28 sept. 1957.

22. HNRB, 11,24 oct. 18,27 nov. 11 déc. 1956, HNRB, 12 janv., DGB, $\mathrm{I}^{\text {er }}$ mars. 23 avril, $Z Z R B, 7$ juil., $H N R B, 11,31$ juil., 4, 6, 7 août. $R M R B, 7$ août, $H N R B, 10,11,13,15,17,24,25$. 27 aoùt, $Z Z R B, 9$ nov.. $D G B, 18$ nov., $H N R B, 8$ déc. 1957. 15 juil. $R M R B, 22$ oct. 1958.

23. $H N R B, 27$ nov. 1956.

24. $D G B, 1^{\text {er }}$ mars, $H N R B, 31$ déc. 1957. En décembre 1957, 15000 tonnes de coton restaient dues par les cooperatives sur une production annuelle de 170000 .

25. Sur la réapparition de l'usure à cette époque, voir aussi $H N R B, 3$ sept., $Z Z R B, 30$ sept. et $D G B, 30$ nov. 1957.

26. Quanmian dayuejin de liangshi gongzuo (Le travail céréalier dans le Grand bond en avant global). Zhengzhou, Editions populaires du Henan, 1958, pp. 60-62.

27. Xinhua banyuekan (Bimensuel Xinhua), 1958, $\mathrm{n}^{\circ} 4$, pp. 127-128. Nous considérons que, de par sa nature bureaucratique et de par sa définition géographique. la profession de cadre est une profession urbaine.

28. Voir par exemple dans $H N R B$, $1^{\text {er }}$ oct. 1956, une description des départs pour la ville des jeunes scolarisés des environs de Xinxiang.

29. $L Y R B, 10.29,30$ mai. $H N R B, 4$ août, ZZRB, 16 nov. 1957.

30. En tout cas. nos sources ne livrent pas de données chiffrées d'une valeur comparable à celles dont font état C. Howe pour Shanghai (Employment and economic growth in urban China, 1949-1957, Londres. Cambridge University Press, 1971), et E. Voge1. pour Canton (Canton under communism).

31. Voir par exemple $H N R B, 4$ août 1957.

32. HNRB, 25 sept. 1957.

33. $L Y R B, 17$ mai et $H N R B, 4$ août 1957.

34. Sur les problèmes d'approvisionnement dans les villes henanaises, voir $H N R B, 16$ juil.. 16 aoùt. $Z Z R B, 15.21$ sept.. $H N R B, 31$ oct. 26 nov. 1956, $D G B, 20$ fév., $L Y R B, 17.28$ mai. 7 juin. $Z Z R B, 29$ oct. 1957.

35. La consommation de porc, par exemple. est passée à Zhengzhou. la capitale henanaise (qui comptait environ 700000 habitants). de 32000 têtes pendant toute l'année 1953 à 39000 durant le premier semestre de 1956, et la consommation de sucre de 794 tonnes en 1952 à 2695 en 1956 (ZZRB, 7 sept. 1956 et 18 juil. 1957).

36. Voir $Z Z R B, 4$ juin. 18 juil., 4 sept. 1956. $L Y R B, 25.27,29.7$ juin, $H N R B, 14$ juil., $Z Z R B$. 16 juil.. $H N R B, 4$ aoút. 18 oct.. $Z Z R B, 23$ oct., 10. 28 nov. 1957.

37. Ainsi, en juin 1957, quarante-quatre familles occupaient des logements vides de lusine mécanique de Zhengzhou ( $Z Z R B, 16$ juil. 1957) et plusieurs familles de cheminots transférées de Xuchang campaient dans les couloirs des logements ouvriers de la gare de Zhengzhou (ZZRB. 23 oct. 1957). 


\section{LA CHINE CONTEMPORAINE}

38. HNRB, 4 sept. 1957.

39. On consultera cependant un rapport du président de la cour suprême de la province (HNRB, 25 août 1957).

40. $H N R B, 4$ juil. 1958.

41. ZZRB, 23 oct. 1957.

42. $L Y R B, 25$ mai, $Z Z R B, 10,16$ oct.. HNRB, 10 déc. 1957.

43. $Z Z R B, 24$ nov. 1957.

44. Voir par exemple $H N R B, 26$ mai 1957.

45. A lautomne 1956. $10 \%$ des membres du Parti dans la région spéciale de Luoyang attendaient d'ètre titularisés (HNRB, 25 oct. 1956). Au Quotidien du Henan, entreprise pourtant politique par excellence. seulement douze personnes reçurent leur carte du Parti entre 1949 et 1957 (HNRB, 26 mai 1957).

46. $H N R B, 9,17$ juin. 3, 9, 10, 21 août 1956, LYRB, 31 mai, $H N R B, 9$ juin. ZZRB, 13, 18. 31 juil., $H N R B, 6$ août 1957.

47. Parmi les 265 démobilisés qui s installèrent dans le district de Yichuan au cours du premier semestre de 1957. 245 se marièrent ou se remarièrent avec l'aide des autorités (HNRB, 6 aoút 1957).

48. $L Y R B, 17.25$. 30 mai, $H N R B, 2,4$ juin. $L Y R B, 6$ juin, $H N R B, 22$ juin. $Z Z R B, 31$ juil.. $H N R B, 8,16,17$ août. $R M R B, 27$ aoùt. $Z Z R B, 23$ oct. 1957.

49. Les femmes de cadres étaient souvent détestées pour leur prétention. Cf. $H N R B, 2,4$ juin. $Z Z R B, 4$ oct. $H N R B, 29$ déc. 1957.

50. $L Y R B, 14,17$ mai, ZZRB, 22 mai, $L Y R B, 27$ mai, $H N R B, 2$ juin. $L Y R B, 6$ juin. $H N R B$, 8 juin 1957

51. Sur ce privilège en matière d'information, voir surtout $L Y R B, 23$ mai, HNRB, 24 mai, 22 juin 1957.

52. $H N R B, 24$ mai. $L Y R B, 6$ juin 1957.

53. $H N R B, 2,4$ juin. $L Y R B, 6$ juin 1957.

54. $H N R B, 31$ janv. $L Y R B, 1^{\text {er }} .13$ mai, 7 juin, $Z Z R B, 20$ juil., 15 sept. 1957.

55. Dans le textile, un ouvrier gagnait en moyenne 70 yuan par mois dès la première année. Mais dans la construction de base le salaire mensuel moyen, après quatre ans d'ancienneté. ne dépassait pas 50-60 yuan.

56. Voir notamment $H N R B, 12$ janv., 14 mai. 31 août. $Z Z R B, 3.21$ sept. 1957.

57. Dans certains cas, la désaffection à leur égard était presque totale. Ainsi. $95 \%$ des cotisations étaient impayées dans une usine de Luoyang ( $L Y R B, 31$ mai 1957).

58. $D G B, 3$ fév., $L Y R B, 24$ mai. ZZRB, 28 oct. 1957.

59. $H N R B, 11$ sept. 1957. Pour un exemple similaire, voir $H N R B, 13$ juil. 1957.

60. $H N R B, 15$ juin. 15 nov. 1956, LYRB, 7 juin 1957.

61. $Z Z R B, 2$ mai, $H N R B, 31$ mai 1957

62. $Z Z R B, 22$ mai. 21 sept. $L Y R B, 22.30 .31$ mai. $H N R B, 24$ mai 1957.

63. $Z Z R B, 19$ juil., $H N R B, 3$ août, 6, 8, 13, 18 sept., 30 nov. 1956, 10 janv.. $L Y R B, 14,17,22$. 31 mai. $Z Z R B, 13$ juil.. $H N R B, 24$ sept. 1957. Renmin jiaoyu (Éducation populaire), 1957. no 9. pp. $27-29$.

64. HNRB, 18 oct. 1956, LYRB, 29, 31 mai 1957, Renmin jiaoyu (Éducation populaire), 1957. $\mathrm{n}^{\circ} 5$, pp. 15-16 et $\mathrm{n}^{\circ} 9$. pp. 27-29.

65. $Z Z R B, 22$ mai, $L Y R B, 24,27,30$ mai, 6, 7 juin 1957.

66. Il ny aurait eu au total que 1600 retraits effectifs dans toute la province mais, à Zhengzhou, le nombre des artisans individuels fut multiplié par neuf (ZZRB, 29 oct. 1957). Voir aussi $L Y R B, 29$ mai, ZZRB, 3 sept. 1957.

67. $Z Z R B, 30$ nov. 1957. L'une d'entre elles disposait de dix employés et d'un capital de 2000 yuan (ZZRB, 7 sept. 1957). Cf. aussi $Z Z R B, 25$ sept. 11.24 oct. 1957. 
68. $L Y R B, 23$ mai. $D G B, 5$ août. $Z Z R B, 14,21$ sept., 24 oct. 11. 14.19 nov. 1957.

69. $L Y R B, 29$ mai. $H N R B, 6$ juin. 24 aoùt. 12 sept.. 28 déc. 1957.

70. HNRB, 17 mai. 6 juin. RMRB, 9 août 1957. Xinxiang ribao (Quotidien de Xiaxiang), 20 avril 1958.

71. Cette proportion est celle des habitants privés de leurs droits civiques dans neuf villes et sept districts ruraux à l'automne 1956 (HNRB, 29 nov. 1956).

72. Voir par exemple $R M R B, 7$ juil. 1957 (cas d'un petit proprietaire foncier).

73. HNRB, 16 juil. 1956. 29 aoüt 1957, ZZRB, 11 sept. 27 nov. HNRB, 30 nov. 1957.

74. $H N R B .6$ juin 1957 .

75. ZZRB, 21 mai, $L Y R B, 23.30$ mai. 7. 12 juin 1957.

76. $L Y R B, 10$ mai, $Z Z R B, 16$ nov. 1957.

77. ZZRB, 4 oct. 1956. HNRB, 11 janv., LYRB, 29 mai 1957.

78. Voir par exemple $Z Z R B .21$ nov. 1956.

79. Pour le seul mois de juillet 1956. voir ZZRB, $1^{\text {er }}, 4,5,12,21$ juil. 1956.

80. Mais d'autres éléments interviennent, qui permettraient de distinguer entre les différentes crises sociales : le rapport entretenu, à un moment précis. par le régime avec ses propres objectifs de transformation : la composition sociologique de lopposition : ses objectifs idéologiques : la nature et la gravite des difficultés qui ont provoqué la crise sociale. A propos de la contestation survenue dans l'hiver 1978 et de ses rapports avec les exemples précédents. voir l'article de G. Padoul dans Projet, février 1979. pp. 159-169.

81. C'est sans doute le plus grave reproche que l'on puisse faire à l'ouvrage, par ailleurs excellent. de R. MAC FARQHAR. The origins of the cultural revolution. Londres. Oxford University Press. 1974: il se préoccupe presque exclusivement des luttes de faction au sommet. Et c'est le principal merite du petit livre de Siwitt Aray (Les Cent fleurs. Chine 1956-1957. Paris, Flammarion. 1973. 186 p.) que davoir mis en rapport les phénomènes sociaux et politiques. 